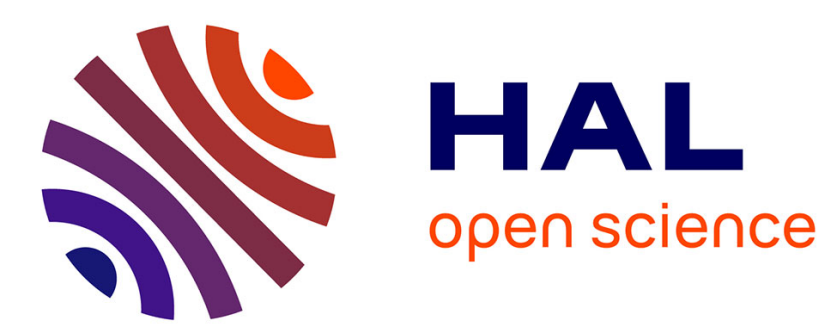

\title{
Recent TEM studies of precipitate growth mechanisms
}

\author{
K.H. Westmacott, U. Dahmen
}

\section{To cite this version:}

K.H. Westmacott, U. Dahmen. Recent TEM studies of precipitate growth mechanisms. Revue de Physique Appliquée, 1986, 21 (12), pp.757-773. 10.1051/rphysap:019860021012075700 . jpa00245497

\section{HAL Id: jpa-00245497 https://hal.science/jpa-00245497}

Submitted on 1 Jan 1986

HAL is a multi-disciplinary open access archive for the deposit and dissemination of scientific research documents, whether they are published or not. The documents may come from teaching and research institutions in France or abroad, or from public or private research centers.
L'archive ouverte pluridisciplinaire HAL, est destinée au dépôt et à la diffusion de documents scientifiques de niveau recherche, publiés ou non, émanant des établissements d'enseignement et de recherche français ou étrangers, des laboratoires publics ou privés. 
Classification

Physics Abstracts

$81.30 \mathrm{M}$

\title{
Recent TEM studies of precipitate growth mechanisms
}

\author{
K. H. Westmacott and U. Dahmen \\ National Center for Electron Microscopy, Materials and Molecular Research Division, University of \\ California, Berkeley CA 94720 , U.S.A.
}

(Reçu le 2 juin 1986, révisé le 11 août 1986, accepté le 26 août 1986)

\begin{abstract}
Résumé. - Des exemples récents d'étude par microscopie électronique en transmission sont analysés dans cette revue. L'accent est mis sur l'identification (i) de la nature des modifications de structure intervenant lors des transformations, (ii) des défauts cristallins et des modes de déformation nécessaires à la croissance du précipité dans la phase mère. Un classement des réactions de précipitation est effectué selon les mécanismes de déformation mis en jeu. Des modèles simples permettant de prévoir la morphologie des précipités sont précisés dans chaque cas. Des éléments de discussion concernant la nature de l'interface entre la matrice et un précipité quelconque en plaquette sont également fournis. Les effets de la symétrie cristalline sur les distributions de forme sont brièvement abordés.
\end{abstract}

\begin{abstract}
In this review recent results of transmission electron microscopy studies of precipitation in a number of model binary alloy systems are presented. Emphasis is placed on identifying (i) the nature of the structural change occurring during the transformations, and (ii) the crystal lattice defects and deformation modes required to accommodate the growing precipitate in the parent phase. Precipitation reactions are classified in terms of their transformation strains, and simple predictive models for the precipitate morphology are developed for each case. Brief discussions are also given on the nature of a general plate precipitate interface and the effect of crystal symmetry on morphological distributions.
\end{abstract}

\section{Introduction.}

It lies beyond the scope of the present article to present a comprehensive review of the large number of precipitation reactions that have been studied. Instead we will highlight a few facets in an attempt to establish a relatively simple framework of underlying principles. By focusing on the structural aspect of phase transformations and by using simple binary metal alloys as model systems, it is possible to paint a systematic picture in which precipitation reactions are characterized by their transformation strain and their mode of strain accommodation in the matrix crystal. In this picture even complex precipitation processes are pieced together from basic building blocks such as the dilatational and deviatoric components of the transformation strain and their accommodation by such defects as vacancies or shear dislocation loops. This approach takes into account kinetic and thermodynamic aspects only insofar as it assumes that a reaction will not proceed unless thermodynamically favorable and kinetically feasible. Thus we will not try to understand at what temperature or how fast a reaction goes, but what are the possible shapes, orientations, interface structures and distributions of precipitates forming under a well-defined set of constraints. This structural focus allows parallels to be drawn between widely different types of alloy system such as interstitial (e.g. Pt-C) substitutional (e.g. Al-Cu) and mixed interstitial/substitutional alloys (e.g. Mo-Hf-N).

The nucleation and growth of precipitates occurs on a scale of $\mathrm{nm}$ to $\mu \mathrm{m}$, and the work reviewed here is based primarily on TEM observations. The mechanisms of contrast formation in the TEM allow a quantitative determination of habit planes, displacement vectors, dislocation content or interface structure. By in situ observation in a high temperature specimen stage it is possible to deduce growth mechanisms directly and even to set up local nonequilibrium concentrations of point defects by electron irradiation in an HVEM.

An alloy far from equilibrium, for example after rapid quenching, can follow many different paths on its return to equilibrium. Its final microstructure depends on the path chosen which in turn depends on the lattice defects available to assist in the formation of the equilibrium phase. In the absence of suitable defects initial phase separation may occur by the formation of metastable intermediate phases (often with a structure approximating that of the more stable phase). 
The focus of this review is on the different ways in which systems can make use of lattice defects on their road to equilibrium. We have drawn heavily on recent research in our own laboratory, and it must be reiterated that a comprehensive review of the work of others has not been attempted.

In the structural view taken here the formation of a precipitate is equivalent to a change in crystal structure and orientation of a volume of material enclosed in the matrix crystal. Assuming the structural change is homogeneous it can be described by a transformation tensor $A$. Any such tensor can be decomposed uniquely into a pure rotation $R$, a pure shear $S$ and a pure dilatation $V$ :

$$
A=R S V \text {, }
$$

or for small strains

$$
a=r+s+v
$$

where $a=A-I$ and $I$ is the identity matrix. Each component of the transformation strain plays a special rôle in the formation and accommodation of the new structure.

The volume charge $v$ can be accommodated either elastically, or plastically by punching out prismatic dislocation loops, by cracking or through excess vacancies or interstitials. The pure shear $s$ requires elastic shear, twinning, faulting, cracking or slip, for its accommodation. The rotation $r$ describes the orientation relationship between precipitate and matrix and depends on the mode and extent of accommodation of the pure shear $s$. The sum of the pure shear $s$ and the pure dilatation $v$ is the pure strain $\varepsilon$ and depends on the lattice correspondence chosen to relate the two lattices. If the principal strains of $\varepsilon$ are of mixed sign then the orientation relationship $r$ usually adds the necessary lattice rotation to produce an invariant line. If in addition, one principal strain of $\varepsilon$ is zero then a rotation $r$ can produce an invariant plane strain. These criteria have a strong bearing on the precipitate morphology and are therefore used in the presentation of the experimental evidence. A further criterion used is the volume change $v$ which also has a strong effect on the morphology ; transformations with $v \simeq 0$ are dominated by shear strain accommodation while those with $v \gg 0$ depend on volume accommodation. Elastic and plastic deformation are fundamentally different but there are some basic links between elastic and plastic yielding which are brought out by modelling the loss of coherency as a simple tensile test.

One direct consequence of the principles outlined here is that for strains of unmixed sign, precipitate dimensions are inverse to the principal strains ; e.g., one zero strain will give rise to a needle shape, two small and one large strain to a plate, and three small equal strains to a sphere or cube.

\section{Invariant plane strain transformations.}

Some of the structurally-simplest phase transformations are encountered in alloy systems where precipi- tate plates form on low-index habit planes. This morphology arises because a good match between the atomic spacings in the precipitate and the matrix exists in the habit plane. Considerable lateral growth of the plate is possible before the developing strain from residual mismatch inhibits further growth. Many alloys of fundamental and practical interest are characterized by an invariant plane transformation strain (IPS).

2.1 Al-Ag. - One of the best known examples of this type is found at the aluminum-rich end of the Al-Ag phase diagram where plates of the hcp $\gamma^{\prime}$ phase precipitate from the supersaturated solid solution on the close-packed $\{111\}$ Al matrix planes. Early progress in understanding the mechanism of precipitate growth normal to the habit plane was made more than two decades ago $[1,2]$. Because the atomic volume of the $\gamma^{\prime}$ phase differs from the matrix by only $\sim 1.5 \%$ it was proposed that thickening of the hcp plate in the fcc matrix i.e. growth normal to the habit plane, could be structurally accomplished in a simple manner. If Shockley partial dislocations are able to nucleate readily on the broad faces of a precipitate, the well-known fact that the $A B C A B C$ fcc stacking can be converted to ABABAB hcp stacking by propagation of a Shockley partial on every other $\{111\}$ plane, provides the necessary growth mechanism. The predictions of this model of ledge growth were elegantly verified in one of the first systematic applications of TEM to precipitation phenomena [2] and more recently in a detailed study by high resolution TEM [3]. A schematic diagram illustrating the effect of propagating ledges bounded by Shockley partials across the precipitate interface is given in figure 1a, while figure $1 \mathrm{~b}$ shows a TEM image of a $\gamma^{\prime}$ plate in Al-Ag containing growth ledges. This alloy system illustrates in its simplest form the concept of transformation dislocations [4] (or coherency dislocations [5]) in which partial dislocations, in appropriate concentrations, transform the parent phase structure to that of the product ; in this case by altering the stacking sequence normal to the habit plane. The three equivalent Shockley partials in the $\{111\}$ habit plane which provide the correct transformation dislocations may be nucleated in any combinations. However, the lowest energy configuration will occur when the net Burgers vector at the periphery of the plate is zero, i.e. each partial is nucleated in sequence e.g. $\mathrm{A} \delta, \mathrm{B} \delta, \mathrm{C} \delta, \mathrm{A} \delta \ldots$ in Thompson's notation. A close approximation to this ideal configuration has in fact been observed by high resolution structure imaging [6]. An example which shows the partial dislocation arrangement at the precipitate/matrix interface is given in figure 2 . It was also directly shown in this study how an extra precipitate layer was periodically incorporated parallel to the habit plane to counter the volume contraction.

At the other extreme, if the same partial is nucleated repeatedly, the transformation will be accompanied by a shape range. If such a precipitate grows out to a free surface it will give rise to surface 

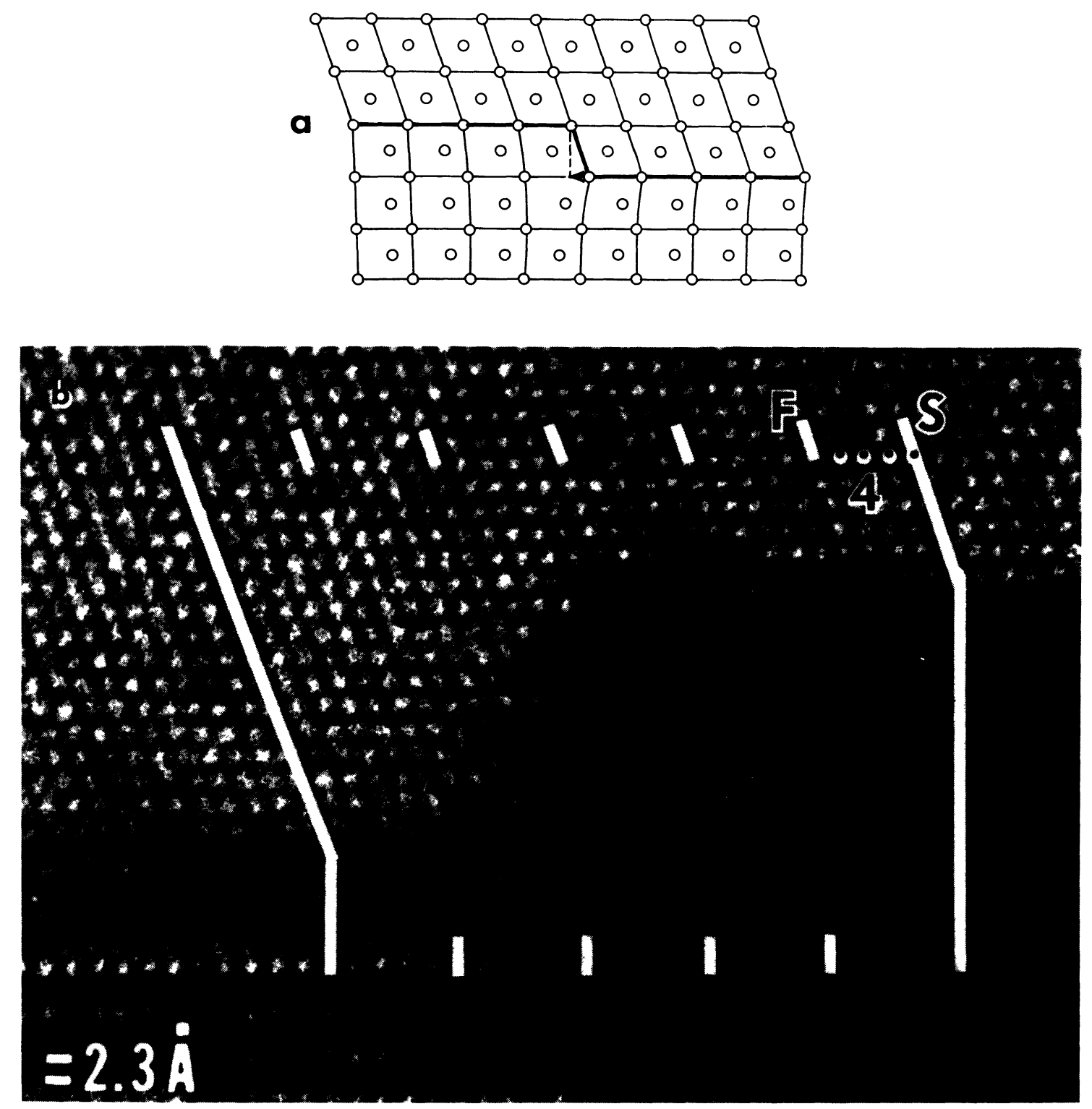

Fig. 1. - (a) Schematic of fcc/hcp interface containing a ledge. The fcc lattice (top) is shown in $\langle 110\rangle$ projection with the interface parallel to a close-packed $\{111\}$ plane. The ledge is associated with a $1 / 6\langle 112\rangle$ Shockley partial dislocation, and its lateral movement requires only small atomic jumps (see arrow), (b) $\langle 110\rangle / /\langle 11 \overline{2} 0\rangle$ HREM image of an approximately ten-plane ledge on the face of a $\gamma^{\prime}$ precipitate, with a Burgers circuit that indicates the presence of four $30^{\circ}$ Shockley partial dislocations associated with the ledge [6].

relief. This aspect, which is a well-known characteristic of martensitic transformations, will be discussed further in a later section.

Until recently it was believed that this simple ledge mechanism would be applicable only to cases of fcc-hcp transformations in which precipitation occurs on $\{111\}$ planes, presumably because of familiarity with the Shockley partial dislocation. Now, however, it is clear that the concept has a much wider validity. By invoking less well-known partial dislocations propagating on other low-index planes the behaviour observed in other IPS systems can be understood. The evidence for these mechanisms will now be reviewed.
2.2 Al-Cu. - The formation of $\theta^{\prime}$ and transition of the metastable phase $\theta^{\prime \prime}$ to $\theta^{\prime}$ in $\mathrm{Al}-\mathrm{Cu}$ alloys provide a simple and graphic example of shear-ledge growth in non- $\{111\}$ plane precipitation. It also serves as the first illustration of the structural, as opposed to a volume accommodation, rôle of vacancies in phase transformations. The discussion is limited to the formation of the $\theta^{\prime}$ phase on $\{001\}$ planes either directly from the supersaturated solid solution or by transition of the metastable $\theta^{\prime \prime}$ phase.

An important step in developing models to describe precipitate growth processes by a ledge mechanism is to identify simple basic lattice correspondences. This is done by considering the various atomic 


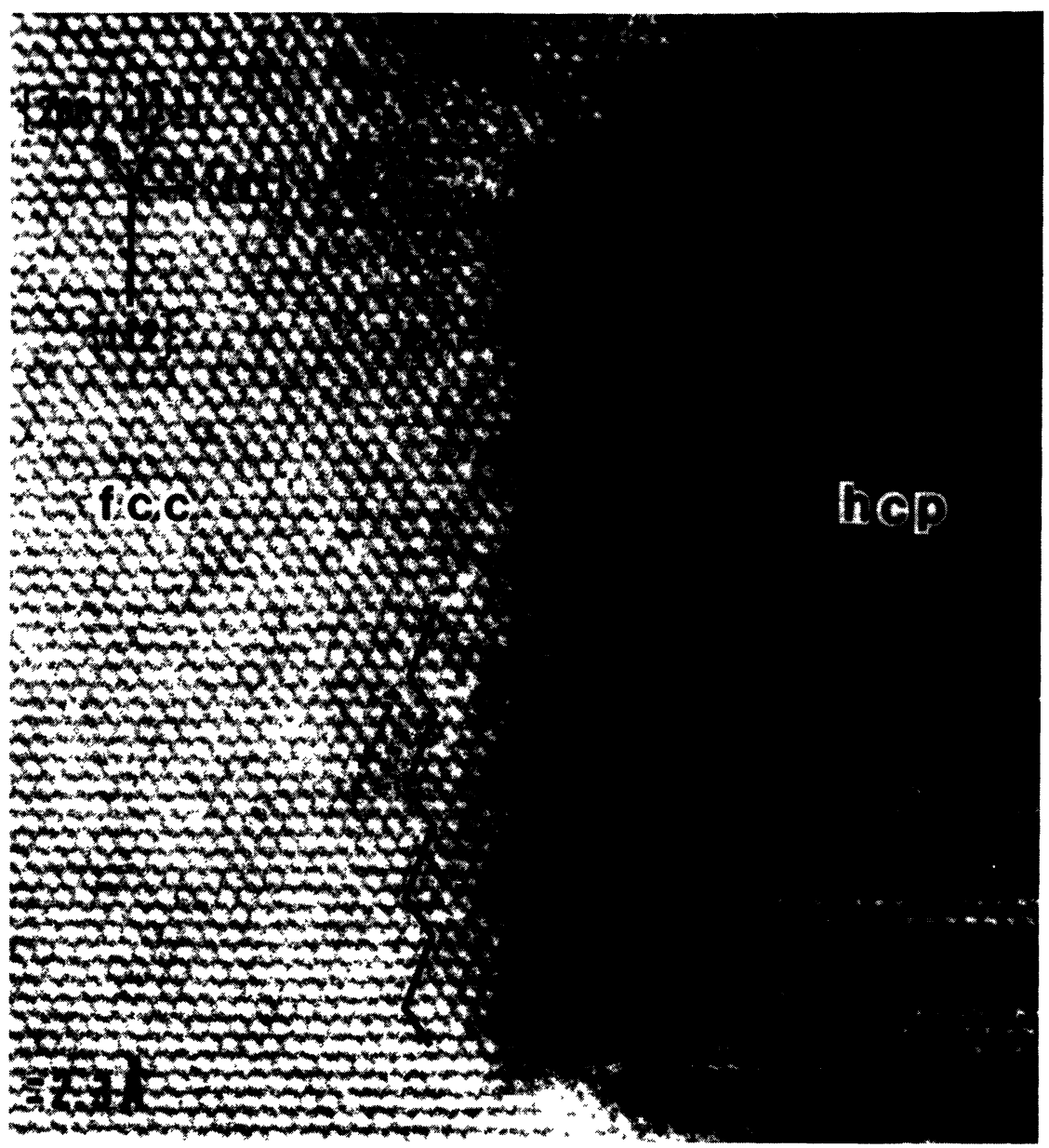

Fig. 2. - HREM image of a precipitate edge in a $\langle 110\rangle / /\langle 11 \overline{2} 0\rangle$ orientation showing that : (1) the nearly planar edge parallels a $\{112\}$ plane in the matrix ; (2) the edge is further faceted along $\{111\}$ matrix planes ; and (3) the $\{111\}$ facets have a six-plane repeating pattern [6].

processes that will take, for example, the structure of the $\theta^{\prime \prime}$ phase into that of the $\theta^{\prime}$ phase [7]. In the case of $\theta^{\prime}$ formation from the solid solution it is assumed that fluctuations in the composition exist such that in local regions layers of copper atoms representing embryonic $\theta^{\prime}$ precipitates are constantly forming.

Some examples of lattice correspondences are given in figure 3. Figure 3(a) shows, in an [010] projection of the lattice, the simplest non-conservative process whereby a $\frac{1}{2}$ unit cell of $\theta^{\prime}$ can form. If a $\frac{1}{2}[100]$ shear partial dislocation is piopagated on a (001) plane it translates the ABABA (001) stacking to $\mathrm{ABAAB}$ as depicted. If this is followed by the non-conservative diffusion of two copper atoms into the newly-created interstices and a small expansion of the lattice in the [001] direction as shown, a half unit cell of $\theta^{\prime}$ is formed. If the same shear occurs over four (001) planes, $\mathrm{AB}^{\prime} \mathrm{AB}$, of the matrix, where $B^{\prime}$ represents a pre-existing layer of copper atoms (see Fig. (3b)), the stacking sequence is changed to $\mathrm{AB}^{\prime} \mathrm{AA}$. Completion of a full $\theta^{\prime}$ unit cell now requires only a shuffle of half of the $B^{\prime}$ layer atoms to the new interstices, accompanied by a slight contraction.

An important point to note is that both processes shown in figure 3(a) and (b) give rise to a shape change (by virture of the shear). Two advantages accrue if the units are taken in the combinations shown in figure 3(c) and (d). First, there is no shape change since the net shear is zero (the structural change can be equivalently considered as due to propagation of a $\frac{1}{2}$ [100] partial dislocation dipole at the levels indicated by an arrow). Secondly, the strains in the $c$ direction normal to the precipitate plate are reduced. Based on these considerations it was proposed [7] that the lowest strain energy growth sequence for $\theta^{\prime}$ from solid solution would be one in which the steps shown in figure 3(d) and (c) occurred consecutively. Fortunately, an accurate set of experimental observations on $\theta^{\prime}$ plate thicknesses had already been made using high resolution electron microscopy [8]. These showed good agreement with the predicted plate thickness of sequence $2,3 \frac{1}{2}$, 


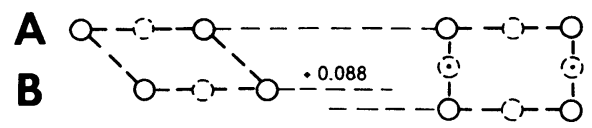
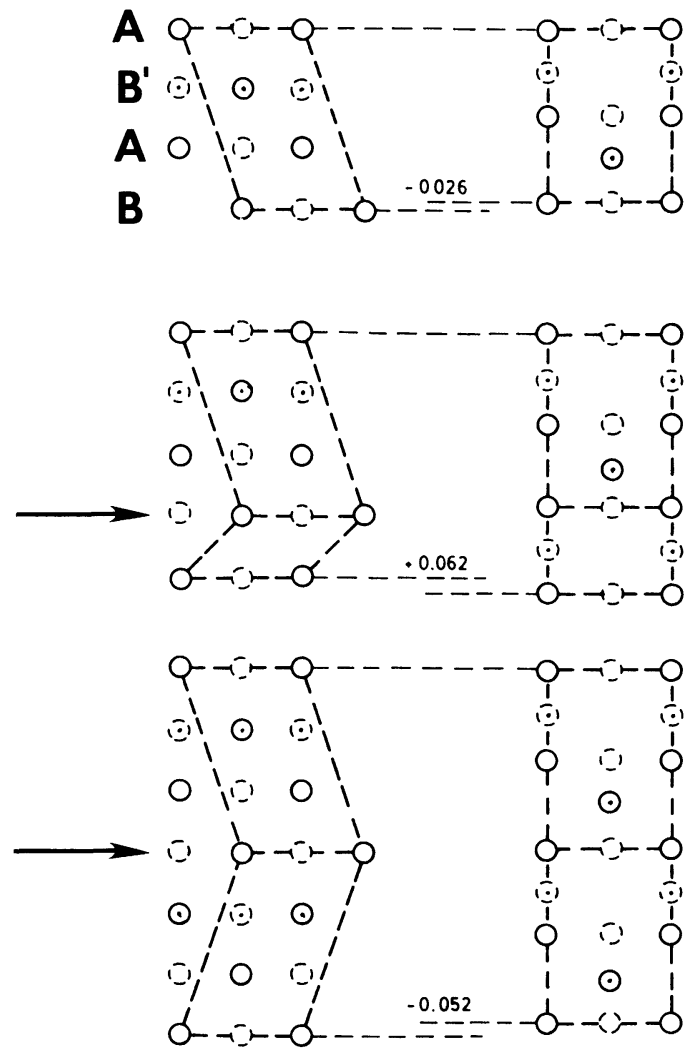

Fig. 3. - Simplified [010] projections of basic (a) nonconservative and (b) conservative units for the f.c.c. to $\theta^{\prime}$ transformation and (c), (d), their combination with opposite shear components. The expansion or contraction component in $\mathrm{nm}$ is shown for each unit.

$5 \frac{1}{2}, 7, \ldots$ unit cells. Significantly, no instances of single unit cell thicknesses were reported, supporting the contention that a net shape change is an unfavorable process.

The $\mathrm{Al}-4 \% \mathrm{Cu}$ alloy has also provided direct evidence that vacancies can fulfill an important structural rôle in phase transformations even when the accompanying volume change is negative. This alloy system has been extensively studied by TEM and it is very well known that the quench-aging treatments that are used to produce the $\theta^{\prime \prime}$ phase also give rise to a high density of perfect prismatic vacancy loops (or helices if the specimen is concurrently deformed). It has not been appreciated, however, that in addition to enhancing diffusion prior to loop formation, the vacancies sequestered in the loops could assist a phase transformation at higher temperatures. This was demonstrated by in situ hot stage experiments in which it was observed that the shrinkage of loops at $180-200^{\circ} \mathrm{C}$ was accompanied by the formation of $\theta^{\prime}$ particles from the $\theta^{\prime \prime}$ phase. Conversely, in the absence of dislocation loops the $\theta^{\prime \prime}$ dissolved and the copper precipitated at the foil surfaces as the equilibrium $\theta$ phase.

A schematic diagram illustrating how vacancies could initiate the transition of $\theta^{\prime \prime}$ to $\theta^{\prime}$ is given in figure 4 . The generally accepted structure of $\theta^{\prime \prime}$ is one in which layers of copper atoms precipitated on $\langle 001\rangle$ planes are separated by three layers of aluminum. The unit cell is usually depicted as the five layer structure shown in figure 4(a). If instead the layers are considered in the equivalent sequence of figure 4(b) it is easy to see how the condensation of a layer of vacancies (liberated from the shrinking loops) at the level indicated, followed by the usual shuffle of half the copper atoms from the nearby layer into the new interstices, leads directly to formation of the familiar fluorite structure of the

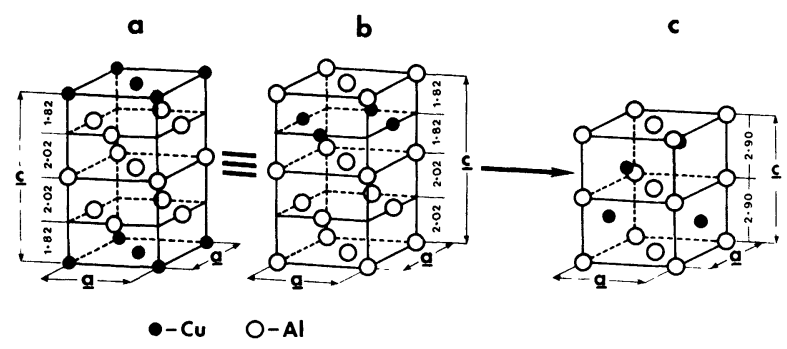

Fig. 4. - Schematic diagram showing the transition of $\theta^{\prime \prime}$ to $\theta^{\prime}$ in $\mathrm{Al}-\mathrm{Cu}$ by the vacancy mechanism. Vacancies from annealing dislocation loops are condensed on a nextnearest Al plane from the copper layer in $\theta^{\prime \prime}$ (b) to form the required A-A-A Al stacking. Formation of the $\theta^{\prime}$ fluorite structure (c) then requires only slight redistribution of the copper atom layer and relaxation of the $\mathrm{Al}$ layer spacings.

$\theta^{\prime}$ phase. In this reaction the vacancies play an essential structural rôle in the transformation since they change the [001] stacking of the initial phase to that required by the final phase. Other examples of this important function of vacancies will be given in the section on Pt-C alloys. A wide variety of precipitate structures can be obtained in $\mathrm{Al}-\mathrm{Cu}$ alloys by varying the heat treatment. In samples quenched directly to a high final aging temperature $\left(\sim 240^{\circ} \mathrm{C}\right.$ ) the $\theta^{\prime}$ is unable to nucleate homogeneously in the matrix but striking heterogeneouslynucleated precipitate arrays are observed $[9,10]$.

The arrays form when $\{110\}$ plane BardeenHerring climb sources are activated and the $\theta^{\prime}$ phase nucleates on the climbing edge dislocations. In this dynamic process the $\theta^{\prime}$ is drawn out behind the dislocation as a lath. Subsequently the lath thickens and breaks up into a row of circular precipitates reminiscent of the annealing behaviour of dislocation dipoles. To investigate the precise way in which the dislocation nucleates the $\theta^{\prime}$ plates, a detailed contrast analysis was performed using the weakbeam imaging technique [11]. The analysis clearly showed that on the (100) segments where $\theta^{\prime}$ was nucleated, the $\frac{1}{2}[110]$ dislocation had undergone an 
usual dissociation into $\frac{1}{2}\langle 100\rangle$ partials, according to the reaction :

$$
\frac{1}{2}\langle 110\rangle=\frac{1}{2}\langle 100\rangle+\frac{1}{2}\langle 010\rangle .
$$

This $\{100\}$ plane dissociation is exactly analogous to the :

$$
\frac{1}{2}\langle 110\rangle=1 / 6\langle 21 \overline{1}\rangle+1 / 6\langle 121\rangle
$$

reaction on $\{111\}$ planes that was found to nucleate the $\gamma^{\prime}$ phase in Al-Ag alloys [1,2]. When further analysis revealed that the trailing $\frac{1}{2}\langle 100\rangle$ dislocation, which eventually bounded the precipitate, was of shear character, the nucleation mechanism became clear. Provided the local chemical composition is favorable, a $\frac{1}{2}$ [100] shear loop on the (001) plane would be the required embryonic structural unit for the formation of a single unit cell of $\theta^{\prime}$ by the process of figure $3(\mathrm{~b})$. Observed changes in the contrast behaviour of the dislocations bounding the thickening precipitates were consistent with this picture ; see figure 5.

This is an intriguing example of the indirect ways in which lattice defects are brought into play in phase transformations. Evidently the temperature is too high for $\theta^{\prime}$ to nucleate directly in the lattice (with or without the aid of vacancies) or on preexisting dislocations. However, by using the vacancies to activate climb sources which propagate dislocations that subsequently dissociate, the system eventually arrives at a lattice configuration where nucleation of the $\theta^{\prime}$ phase becomes favorable.

2.3 Pt-C. - The observation of precipitate structures in this dilute interstitial alloy system [12] provided the first direct, and most striking, evidence of the structural rôle of vacancies in an IPS transformation. By conducting detailed contrast analyses of the precipitate plates at various stages in the growth sequence, the idealized model depicted in figure 6

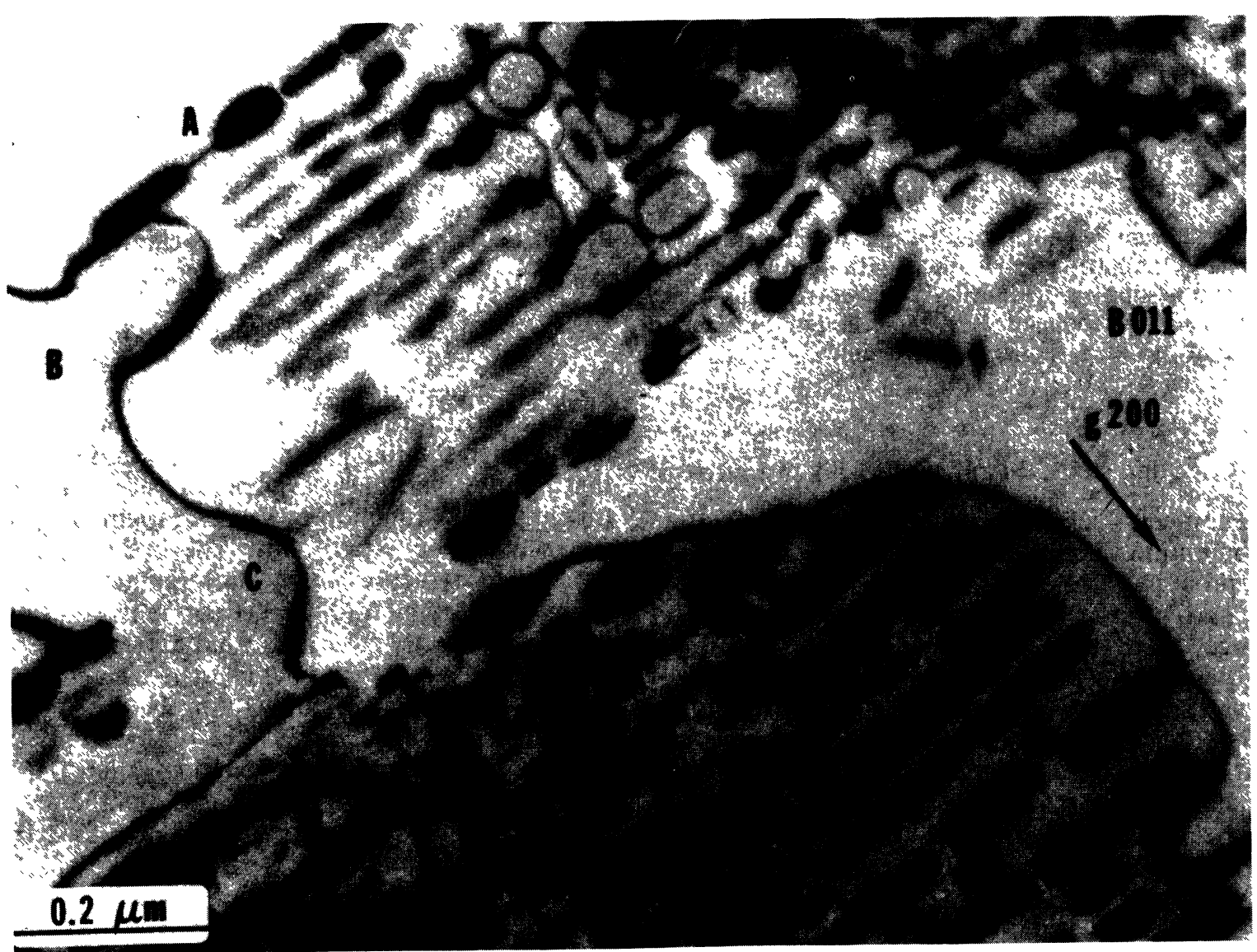

Fig. 5. - (101) colony in [011] projection showing at A the change in contrast of edge-on precipitates corresponding to increasing thickness behind the growth front. At B a single unit cell thick precipitate has formed by dissociating the $\frac{1}{2}[101]$ dislocation at the growth front on the (001) plane into a leading $\frac{1}{2}$ [001] partial (pure edge, invisible) and a trailing $\frac{1}{2}[100]$ partial dipole. At $\mathrm{C}$, the observed contrast is consistent with a two-unit-cell precipitate in which the second cell formed with an opposite shear from the first. 
a

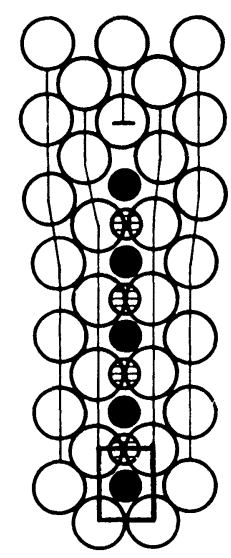

b

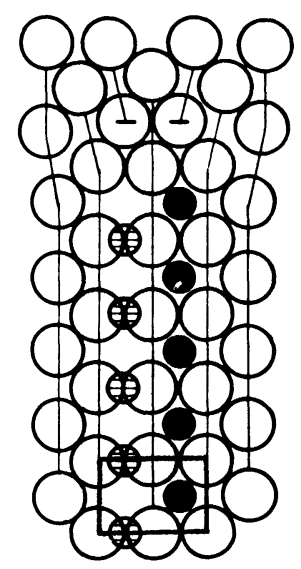

Fig. 6. - Carbide precipitation in Pt occurs first by the coprecipitation of carbon atoms and vacancies on $\{001\}$ planes to form semicoherent GP zones (a). During further aging a second layer of vacancies is condensed on a nextnearest $\{001\}$ plane and half of the carbon atoms " shuffled» over to form the final fluorite structure (b) (isomorphous with $\theta^{\prime}$ ); unit cells shown in heavy outline.

was constructed. Initially, following a quench, semicoherent plates of carbon form on $\{001\}$ planes by the co-precipitation of vacancies and carbon atoms, as shown in figure 6(a). During subsequent aging, the precipitates coarsen and the resulting doublelayer structure of the surviving precipitates is shown in figure 6(b). Its formation mechanism almost parallels that of $\boldsymbol{\theta}^{\prime}$ in $\mathrm{Al}-\mathrm{Cu}$. The initial coprecipitation produces a layer of carbon and AA (001) plane stacking. A second layer of vacancies condenses on a next-nearest (001) plane during aging, resulting in AAA stacking; then small carbon atom shuffles lead to formation of a $\mathrm{Pt}_{2} \mathrm{C}$ carbide isomorphous with $\theta^{\prime}$. This picture was fully confirmed by microdiffraction analyses on individual precipitates using fine electron probes [13].

Several aspects are noteworthy : (1) the precipitates form only in the presence of excess vacancies ; this is because $\mathrm{Pt}_{2} \mathrm{C}$ is $50 \%$ oversize in the $\mathrm{Pt}$ matrix and vacancies are vital in a second rôle of providing volume accommodation ; (2) the observations imply that a large binding energy exists between carbon atoms and vacancies [14]; (3) vacancies can be important not only in substitutional but also in interstitial precipitation reactions.

The essential rôle of vacancies in $\mathrm{Pt}-\mathrm{C}$ is duplicated in substitutional systems when there is a large disparity in the atomic volumes of the parent and product phases. Direct evidence for this was found in the precipitation of pure silicon and germanium plates from solid solution in aluminum where the emergence of the first precipitates and their morphological type was correlated with the vacancy concentration $[15,16]$.

As discussed earlier, the propagation of a ledge across the face of a precipitate plate can occur by

both conservative and non-conservative atomic processes. Examples are the expansion of a partial dislocation shear loop and condensation of a vacancy (or interstitial) platelet, respectively. The Pt-C alloys provided an example where both processes occur together. In figure 7 the plate shown was captured as a second unit cell of $\mathrm{Pt}_{2} \mathrm{C}$ was growing by the ledge mechanism on the first. Since the plate lies on a (010) plane, growth solely by the non-conservative vacancy mechanism would mean all the growth ledges are bounded by dislocations with $\langle 0 k 0\rangle$ pure edge Burgers vectors. Consequently, in the 200 reflection operating in figure 7(a) the plate would be completely out of contrast. In fact, the strong contrast visible at the growing ledge is unequivocal evidence that this dislocation has a shear component of Burgers vector lying in (010). The full analysis shows the ledge is composed of $1 / 4[0 \overline{1} 0]$ and $1 / 4[\overline{2} 10]$ components as shown schematically in figure $7(\mathrm{e})$.
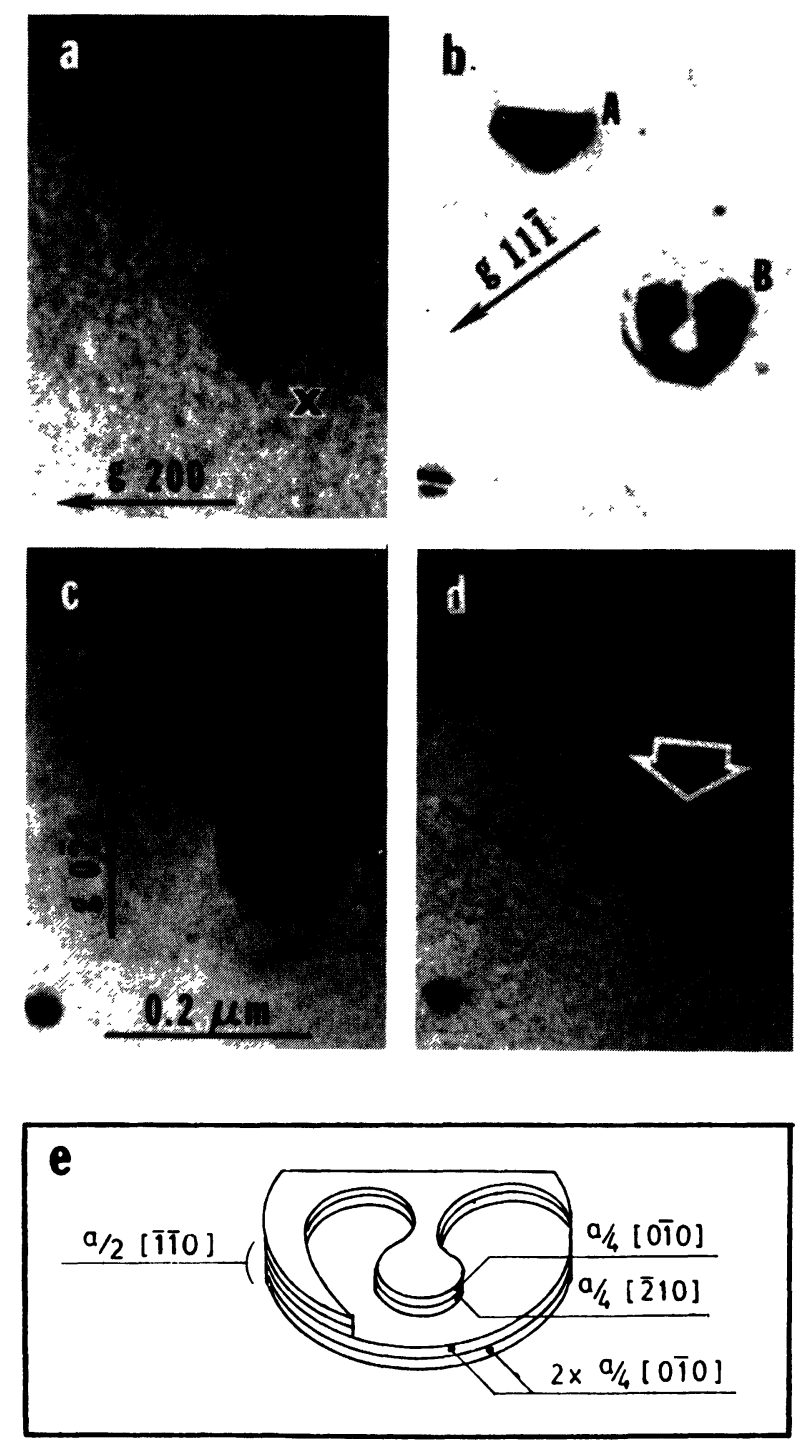

Fig. 7. - Contrast analysis illustrating growth of carbide precipitate in $\mathrm{Pt}$ by conservative and non-conservative mechanisms (micrographs by M. I. Perez). 
2.4 Mo-HfN. - As a final example of a growth process dependent on a supply of vacancies the MoHfN system is considered $[17,18]$. The HfN precipitate has the $\mathrm{NaCl}$ (fcc) structure and is related to the Mo (bcc) structure by the Bain correspondence, hence the precipitation reaction requires no change in the $\{001\}$ stacking sequence. However, there is a $36 \%$ increase in atomic volume implying vacancies are essential for precipitation to occur. In accordance with expectations, plate-shaped precipitates do form on $\{001\}$ and grow by a ledge mechanism, see figure 8. It is clear, however, that the $A B A B A B$ stacking of the matrix $\{001\}$ planes will be preserved during precipitation (as required) only if the vacancies are condensed in double (AB) layers.

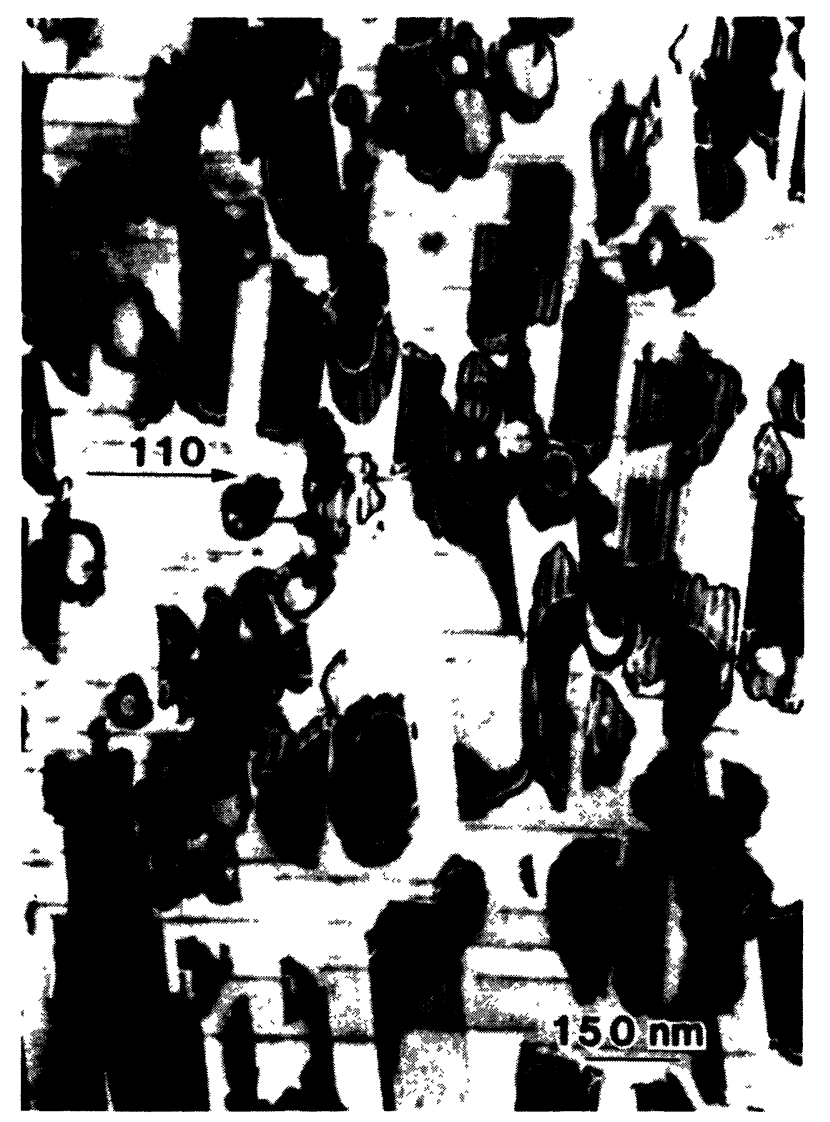

Fig. 8. $-\{001\}$ plate precipitates of $\mathrm{HfN}$ in Mo showing displacement fringe contrast and ledges ; $\langle 110\rangle$ zone axis (micrograph by C. J. Echer).

Structure images obtained on the Berkeley Atomic Resolution Microscope have confirmed this prediction. As seen in the micrograph of figure 9(a), taken in a $\langle 001\rangle$ zone axis, the HfN precipitate contains no stacking faults, and there are extra lattice fringes in the matrix corresponding to the condensation of layers of vacancies. Thus, the observations are in accord with the simple model illustrated in figure $9(\mathrm{~b})$.
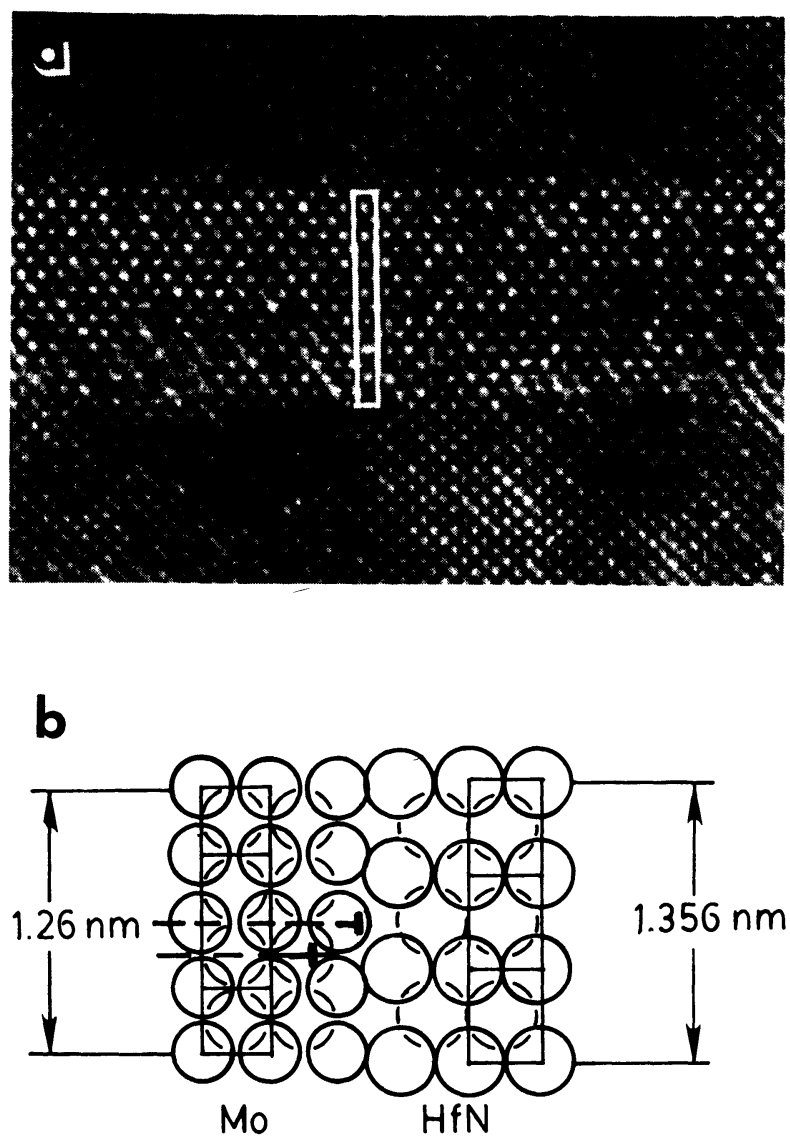

XBL $8311-4637$

Fig. 9. - (a) High resolution micrograph of a single $\{001\} \mathrm{HfN}$ plate is Mo seen edge-on ; (b) schematic diagram of the end of a three-unit-cell thick HfN precipitate plate (in $\langle 110\rangle$ projection) in a Mo matrix (in $\langle 001\rangle$ projection). The two extra planes in the Mo correspond to the condensation of two $\frac{1}{2}\langle 001\rangle$ vacancy loops.

\subsection{GENERAL INVARIANT PLANE INTERFACES. -}

The thickening of IPS plates cannot occur by random attachment of atoms at the interface but proceeds by ledges that sweep laterally across the plane of good fit.

As discussed in the section on Al-Ag, a Shockley partial dislocation changes the stacking of closepacked planes from fcc to hcp and thus bounds a ledge two atomic planes high as illustrated in figure 1(a). The ledge and its associated partial dislocation are inseparable. As an atom crosses the interface it performs a partial jump (indicated by the arrow) that may be expected to have an activation energy different from that of a diffusional jump in the matrix or the precipitate. The glide of a Shockley partial is a succession of such partial jumps and this is in fact identical to the kink-on-ledge mechanism of ledge growth. In the case of $\mathrm{Al}-\mathrm{Ag}$, the glide rate is controlled by the diffusion of $\mathrm{Ag}$ to the ledge. A precipitate that grows by sweeping the same type of 
Shockley partial across every other plane must cause surface relief where it intersects the surface, in the same way that slip bands are formed at the surfaces of deformed crystals.

Consider the case of transformation strain with mismatch described by the dilatational strain $e$ within the close-packed planes in addition to the half-twinning shear of the fcc-hcp transformation. There are two possibilities for the structure and position of the habit-plane. (1) The habit plane is the close-packed plane, as before, and the mismatch is taken up by a set of perfect dislocations (Fig. 10(a)). These dislocations must climb as the interface advances. (2) The habit plane is rotated through an angle $\theta$ related to the shear strain $s$ and the dilatational strain $e$ in the habit plane by :

$$
\tan \theta=e / s \text {. }
$$

This is shown in figure 10 (b) where the interface is seen to lie along a high-index direction $\mathbf{u}$, which is undistorted and unrotated, i.e. an invariant line. When this invariant line direction is resolved onto every other close-packed plane the staggered array of ledges shown in figure 10 (c) is formed. This is the case of an fcc-hcp transformation where every ledge is bounded by the same $1 / 6\langle 112\rangle$ partial dislocation. If instead the invariant line is resolved onto every close-packed plane, the staggered array of figure $10(d)$ results. This is the case of an fcc-bcc transformation with every ledge bounded by the same $1 / 12\langle 112\rangle$ partial dislocation. This kind of array has also been called « structural ledges » [19], and it is apparent from figure 10 that structural ledges differ from growth ledges only by their regular spacing. In a geometrical sense this interface is glissile because it can move conservatively by sweeping the array of partial dislocations across the close-packed planes. As in the case of Al-Ag this will proceed only as the required atoms arrive, and surface relief will result if the dislocations leave the surface.

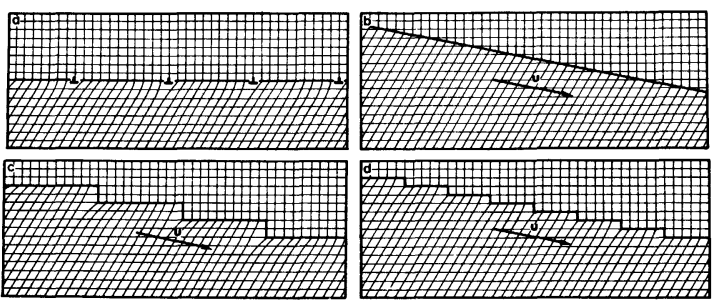

Fig. 10. - Possible interfaces for a transformation described by a shear $s$ coupled with an expansion $e$ in the shear plane. In (a) the expansion $e$ is accommodated by a set of perfect lattice dislocations bounding extra half planes. In (b) the interface lies along an irrational invariant line u and no dislocation accommodation is necessary. In (c) this invariant line is resolved onto every other close-packed plane as in an fcc-hcp transformation. Every ledge is associated with a $1 / 6\langle 112\rangle$ partial dislocation. In (d) the invariant line is resolved onto every close-packed plane as in an fcc-bcc transformation. Every ledge is associated with a $1 / 12\langle 112\rangle$ partial dislocation.
The interfaces shown in figure 10 are two-dimensional sections and simplified cases. A three-dimensional representation of the structural ledges in figure 10 (d) is shown in figure 11. For clarity, the crystal lattices have been omitted. The ledge height is again the spacing of the close-packed planes. As in figure 10 , the partial dislocations at the ledges are not shown. However, there is a set of dislocations lying in the interface parallel to the invariant line $\mathbf{u}$. Their rôle is to accommodate the interfacial mismatch in a direction $v$. Unlike the coherency or transformation dislocations that are associated with the structural ledges these are perfect lattice dislocations.

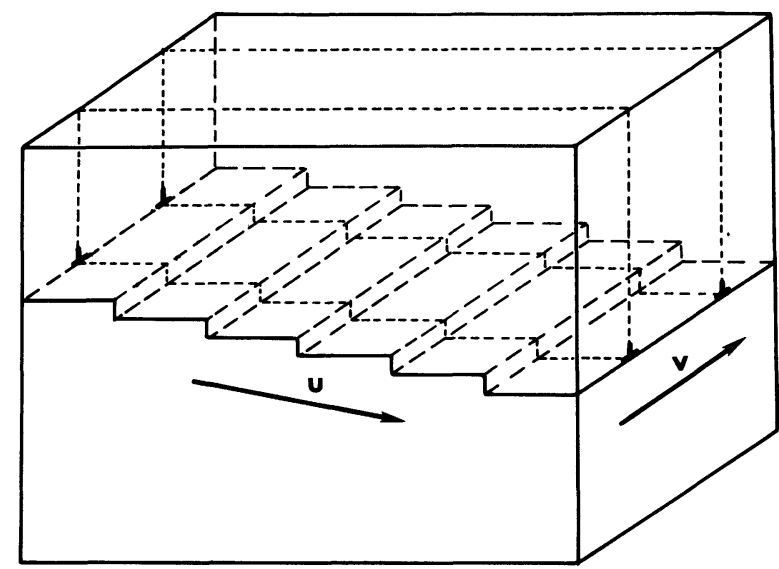

Fig. 11. - The interface of figure 10(d) in perspective with the crystal lattices omitted for clarity. The misfit in the direction $\mathbf{v}$ is accommodated by perfect lattice dislocations indicated by extra half planes parallel to the invariant line u. These dislocations must climb as the interface advances.

The interface in figure 11 illustrations the case of a misfit that is a simple extension in a low-index direction $\mathbf{v}$. The extra half planes of the dislocations are indicated to show that this interface cannot advance conservatively. For the interface to be capable of moving conservatively the misfit dislocations must be able to glide while remaining parallel to the invariant line $\mathbf{u}$. These dislocations must therefore lie along the intersection of their glide plane with the plane of the interface, or habit plane. Such a glissile interface is shown in figure 12 . The misfit dislocation lines are seen to lie in the interface where it intersects their glide planes. This direction $\mathbf{u}$, the invariant line, is once again resolved onto close-packed planes to form ledges and the remaining misfit in the direction $\mathbf{v}$ is a simple shear strain that is accommodated by dislocation shear, as indicated. In general, this direction $\mathbf{v}$ will not be rational either and may therefore be resolved into a second set of structural ledges on low-index planes. Figure 12 shows this feature for an interface similar to that in figure 11. As a further simplification, the upper crystal has been removed to expose the ledge structure of the interface. The crystals on either side could again be fcc and bcc, or any other dissimilar structures. 


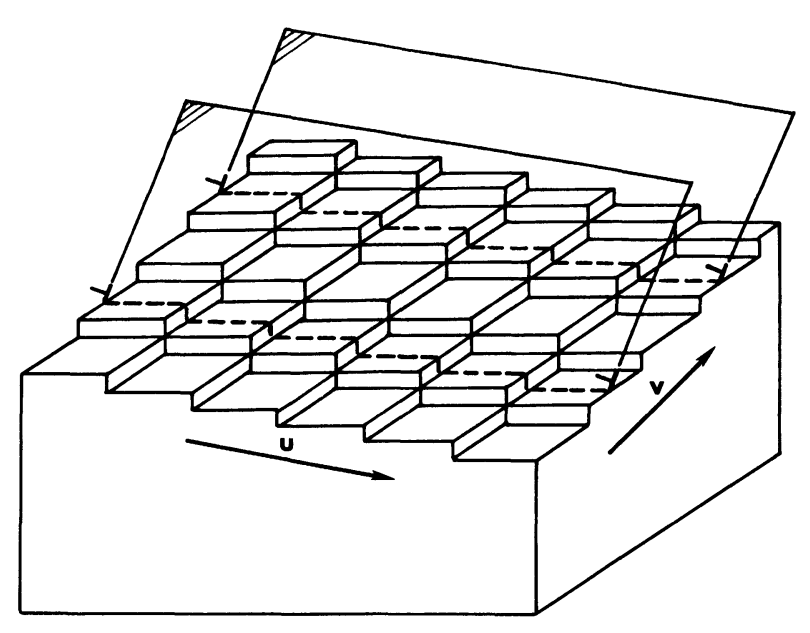

Fig. 12. - A geometrically glissile invariant plane strain interface. In contrast to figure 11 the direction $\mathbf{v}$ is no longer a low index direction and is resolved into structural ledges on low index planes. The misfit (a simple shear) is accommodated by a set of shear dislocations parallel to the invariant line u. Their glide planes are indicated. For clarity the upper crystal has been removed.

Note that this type of interface is not found in any of the simple alloy systems illustrated above where the transformation strain alone leaves a low-index plane undistorted and unrotated. Lattice rotations, dislocation arrays and structural ledges become necessary only when the transformation strain alone is not of the IPS type. In this case it is often found that an invariant line is maintained in the particlematrix interface, and both needles and plates are observed with high-index habits.

\section{Invariant Line Strain (ILS) transformations.}

One of the simplest precipitate morphologies, needle- or rod-like, arises from low misfit along the needle axis. If one of the principal transformation strains is zero the corresponding principal direction is an invariant line and a direction of easy growth for a precipitate. Even if all the principal strains differ from zero but are of mixed sign, an invariant line can be found by adding an appropriate lattice rotation. This is illustrated schematically in two dimensions figure 13(a) and (b), where an unextended line at the intersection of the strain ellipse with the unit circle (a) can be rotated back to become an invariant line (b). The rotation also changes the compressive and dilatational strains (Fig. 13(c)) into shear strains (Fig. 13(d)), so that growth normal to the invariant line depends mainly on shear strain accommodation.

In three dimensions a whole cone of unextended lines is found at the intersection of the strain ellipsoid with the unit sphere and any unextended line can be made an invariant line by the appropriate rotation. In the absence of other constraints, precipitate needles could therefore lie anywhere on the cone of unextended lines. This was indeed found for coherent $\mathrm{Cr}$ needles in $\mathrm{Cu}$ which are distributed on
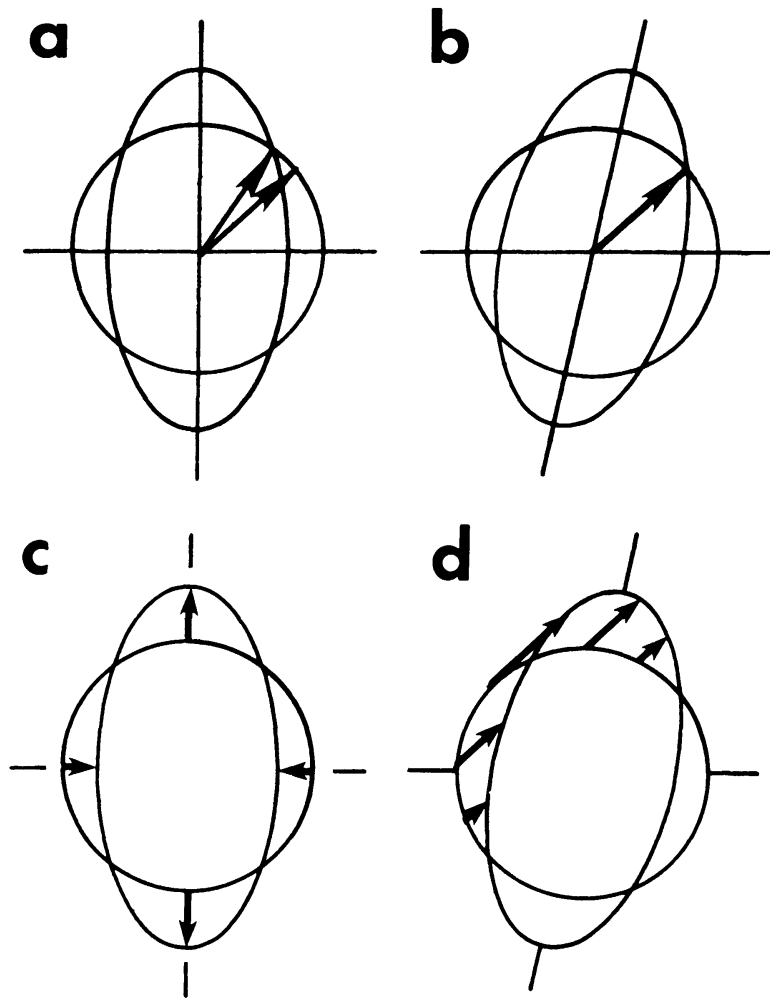

XBL 838-10900

Fig. 13. - (a) Schematic representation of a transformation transforming a circle into an ellipse. The two vectors show an unextended line before and after the transformation. (b) A small rotation makes the unextended line into an invariant line. (c) The same transformation as in (a) with vectors indicating positive and negative strains. (d) The same lattice rotation as in (b) aligns all the strain vectors in one direction approximating a simple shear.

cones centred on the three $\langle 100\rangle$ directions [20]. Their aspect ratio is large and the coherency strains give these needles the appearance of dislocation dipoles. Coherent growth can continue until the stresses that develop normal to the invariant line reach a critical value and the needles lose coherency by nucleating shear loops. This process is most efficient if the needle axis lies on a slip plane, a crystallographic constraint that limits the needles to growth along the direction of intersection between the slip plane and the cone of unextended lines.

3.1 $\mathrm{Fe}-\mathrm{Cu}$ AND $\mathrm{Cu}-\mathrm{Cr}$. - Figure 14 illustrates in stereographic projections the geometry described above for $\mathrm{Fe}-\mathrm{Cu}$ (bcc-fcc) and $\mathrm{Cu}-\mathrm{Cr}$ (fcc-bcc) alloys. The slip planes and their intersections with one cone of unextended lines are shown in (a) for $\mathrm{Fe}-\mathrm{Cu}$ and in (b) for $\mathrm{Cu}-\mathrm{Cr}$. All crystallographically equivalent invariant line directions are shown in figure $14(\mathrm{c})$ and (d) for $\mathrm{Fe}-\mathrm{Cu}$ and $\mathrm{Cu}-\mathrm{Cr}$, respectively. For $\mathrm{Fe}-\mathrm{Cu}$, the invariant lines cluster in two groups of three (open and solid circles) around the $\langle 111\rangle$ directions, whereas for $\mathrm{Cu}-\mathrm{Cr}$ they form clusters of four around the $\langle 110\rangle$ directions. As seen from figure $14(\mathrm{a})$ and (b) this is due to the 

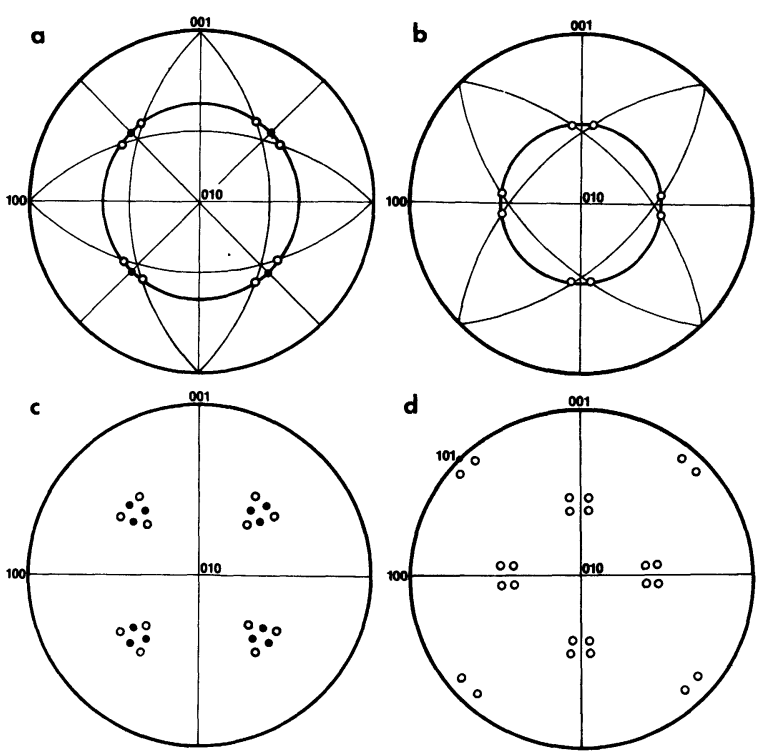

XBL 838-10902

Fig. 14. - Stereograms showing predicted needle directions at slip plane intersections with a cone of unextended lines (a) in $\mathrm{Fe}-\mathrm{Cu}$ and (b) in $\mathrm{Cu}-\mathrm{Cr}$. Only $\langle 651\rangle$ intersections occur in $\mathrm{Cu}-\mathrm{Cr}$ (b) while $\mathrm{Fe}-\mathrm{Cu}$ shows two types of intersection, $\langle 557\rangle$ (open circles) and $\langle 656\rangle$ (solid circles). In (c) and (d) the full set of directions is shown for each of the two alloys when all three cones of unextended lines are taken into account. $\mathrm{Fe}-\mathrm{Cu}$ exhibits two sets of threefold clusters around $\langle 111\rangle$ directions (c) while $\mathrm{Cu}-\mathrm{Cr}$ shows a single set of fourfold clusters around $\langle 110\rangle$ directions.

different slip plane geometry. Experimental results have shown [21] that semicoherent needles in these two alloys indeed lie along the predicted directions, $\langle 651\rangle$ in $\mathrm{Cu}-\mathrm{Cr},\langle 557\rangle$ and $\langle 656\rangle$ in $\mathrm{Fe}-\mathrm{Cu}$. Figure 15 shows a distribution of $\langle 651\rangle \mathrm{Cr}$ needles

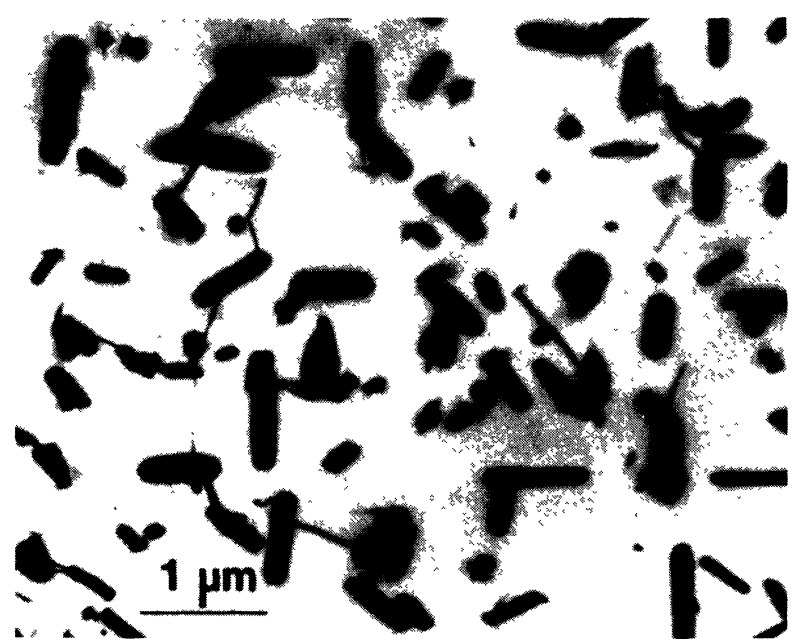

Fig. 15. - Distribution of 24 variants of semicoherent $\mathrm{Cr}$ needles in $\mathrm{Cu}-\mathrm{Cr}$ alloy ; $\langle 001\rangle$ beam direction.

in $\mathrm{Cu}$ seen along a $\langle 100\rangle$ zone axis and figure 16(c) shows a distribution of $\langle 557\rangle \mathrm{Cu}$ needles in $\mathrm{Fe}$ viewed along a $\langle 110\rangle$ zone axis. For comparison, the predicted needle axes and their projected directions are shown in $\langle 110\rangle$ stereograms in figure 16(a) and (b), respectively. This orientation allows a relatively accurate measurement of the angles subtended by many needle directions and it was found that most $\mathrm{Cu}$ needles were of the $\langle 557\rangle$ type with only $\sim 5 \%$ of all precipitates being of the $\langle 656\rangle$ type. Again this can be understood in terms of the more efficient strain accommodation for $\langle 557\rangle$ needles. The transformation strains for these two alloys are large with principal strains of mixed sign but almost no volume change. The resulting precipitates are needles along highindex directions.
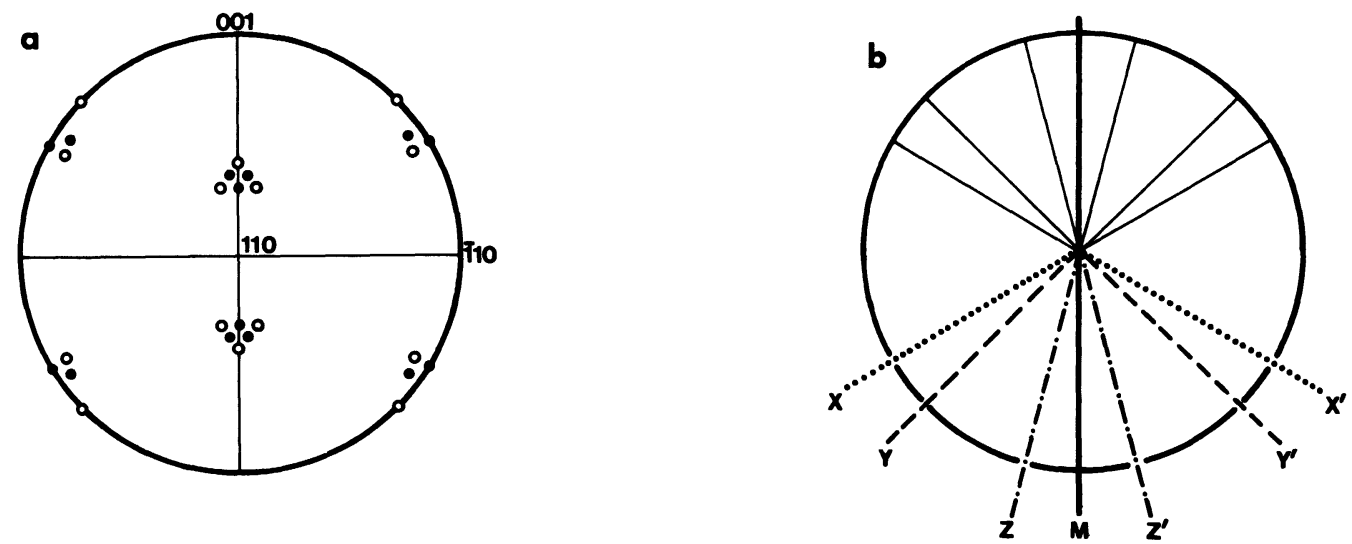

Fig. 16. - (a) Predicted needle directions in $\mathrm{Fe}-\mathrm{Cu}$ in a [110] stereogram with symbols as in figure 14. In (b) the projected directions of the $\langle 557\rangle$ needles (open circles) are marked, $X, X^{\prime}, Y, Y^{\prime}, Z, Z^{\prime}$, and $M$. The $M$ variant lies on the (110) mirror plane which bisects the angles between the other variants, e.g. $Z$ and $Z^{\prime}$. 


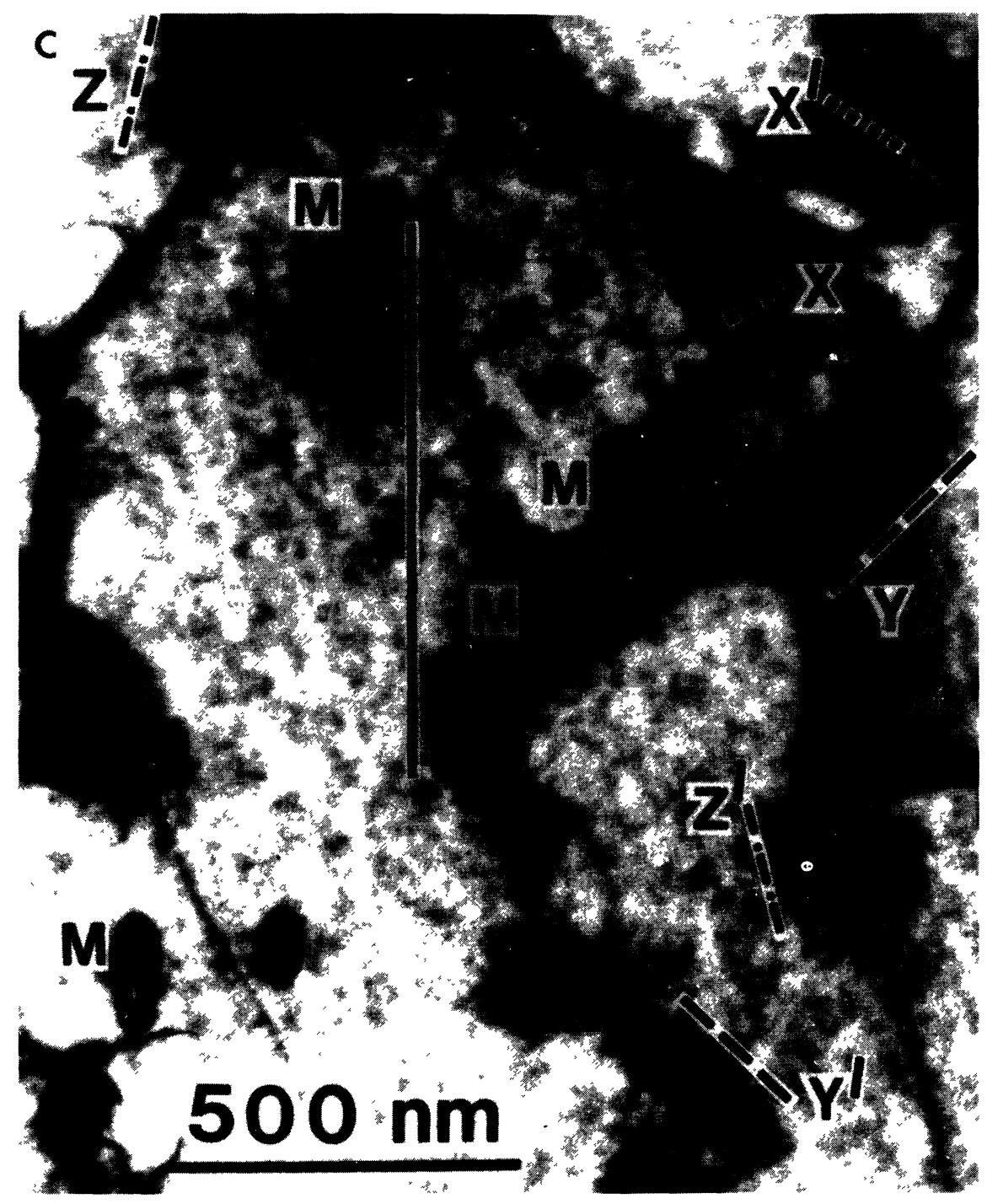

Fig. 16. - (c) Distribution of $\langle 557\rangle \mathrm{Cu}$ precipitates in a [110] zone. The needle directions can be compared directly with those predicted. Note Moiré fringes along precipitates marked $M$. The aspect ratio is low for $Z$ and $Z^{\prime}$ and high for $M$ variants.

3.2 Al-Ge AND Al-Si. - Needle precipitates along low-index directions are found in Al-Ge [22] and Al$\mathrm{Si}[23]$ where one of the principal strains is zero and the volume change is large. As seen in the micrograph in figure 17 the needles in Al-Ge have a high aspect ratio and lie along $\langle 100\rangle_{\mathrm{Al}}$ directions, giving a more crystallographic appearance than the $\mathrm{Cu}-\mathrm{Cr}$ alloy. However, due to the large volume change, vacancies are essential to the precipitation of $\mathrm{Ge}$ in $\mathrm{Al}$, and due to the large crystal structure difference between the parent and product phase, nucleation is difficult. Several lattice correspondences are possible leading to several different orientation relationships and corresponding morphologies. $\langle 110\rangle_{\mathrm{Al}}$ needles and $\{111\}_{\mathrm{Al}}$ plates are frequently found in addition to the $\langle 100\rangle_{\mathrm{Al}}$ needles, and even for the latter needles at least three different orientation relationships have been observed which have only the $\langle 100\rangle_{\mathrm{Al}}$ invariant line direction in common [24].
The difference between them can be seen in the high-resolution micrographs in figure 18 where the different morphologies of the needles in cross-section result from their different orientation relationships with the matrix. It is likely that the twins visible in these particles are due to shear strain accommodation similar to the nucleation of shear loops in $\mathrm{Cu}-\mathrm{Cr}$ and $\mathrm{Fe}-\mathrm{Cu}$ alloys.

3.3 FROM NEEDLES TO PLATES. - Alloy systems with mixed principal strains and large volume change are often found to form plates rather than needles. These plates contain an invariant line direction in the interface commonly visible in semicoherent plates as a set of parallel striations across the precipitate. Figure 19 illustrates this for a $\mathrm{Mo}_{2} \mathrm{C}$ carbide precipitate in a Mo matrix [25]. The striations lie along $\langle 113\rangle$ directions, the intersection of the cone of unextended lines with the slip plane. The 


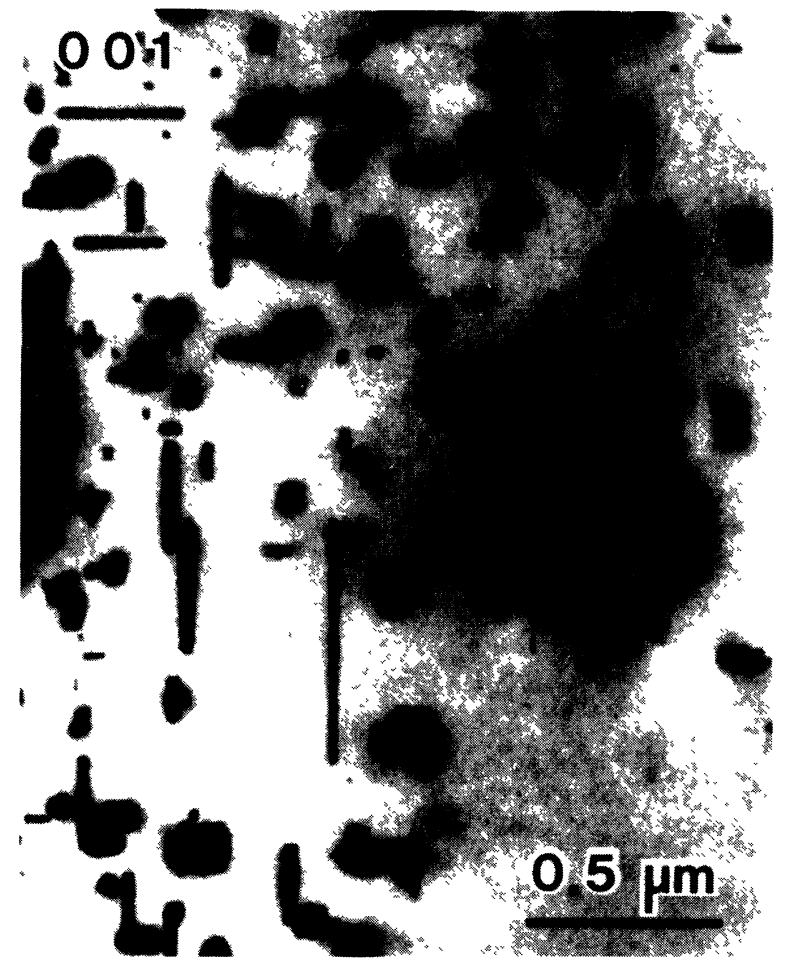

Fig. 17. - Distribution of three $\langle 100\rangle$ variants of Ge needles in Al-Ge alloy ; $\langle 001\rangle$ beam direction.

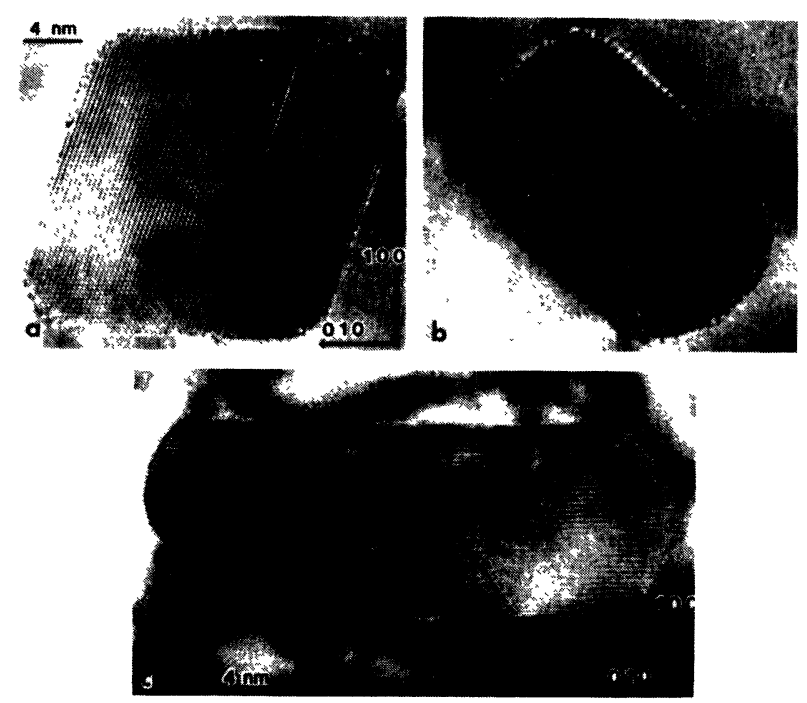

Fig. 18. - High resolution images of Ge precipitates in Al-Ge alloy seen along common $\langle 001\rangle_{\mathrm{Al}} /\langle 110\rangle_{\mathrm{Ge}}$ direction exhibiting different orientation relationships and corresponding shapes (a) shows monoclinic, (b) orthorhombic and (c) tetragonal symmetry.

invariant line condition is therefore identical to the case of needle precipitates and such a plate may be visualized as a parallel array of needles slipped with respect to each other along the slip plane [26].

An alternative view uses the analogy with a tensile test and shows more graphically the connection with coherent precipitate plates and the process of cohe- a
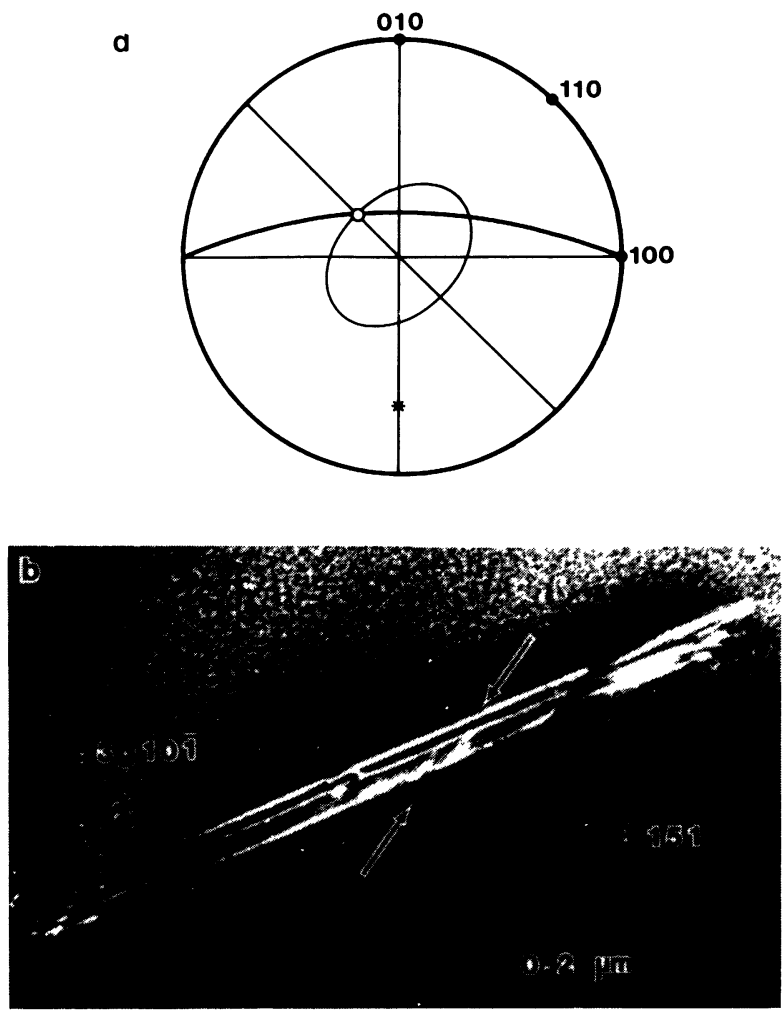

Fig. 19. - Semicoherent plate precipitates in Mo-C alloy (bcc-hcp transformation) ; (a) shows stereogram with elliptical cone of unextended lines, (110) (0001) slip plane, [113] invariant line (open circle) and ( $0 \overline{3} 1)$ habit plane (star) ; (b) shows a TEM image of an inclined $(0 \overline{3} 1)$ plate with interface striations along invariant line direction.

rency loss [27]. Consider a thin coherent plate with mixed principal strains. Oriented for minimum strain energy it contains an invariant line in the interface and its state of stress is dominated by the uniaxial tension in the plane of the plate, normal to the invariant line. The strain energy density is approximately

$$
W=\frac{1}{2} E e^{2},
$$

where $e$ is the principal strain normal to the invariant line and $E$ is Young's modulus. If the crystal is elastically anisotropic, the inclined plate will orient its tensile axis to lie along an elastically soft direction. If the coherency stresses become too high the precipitate loses coherency by plastic deformation. It is most likely to do so on the slip system with the highest resolved shear stress ; ideally with both the slip plane and the slip direction at $45^{\circ}$ to the tensile axis. Thus, under the stress imposed by the constraining matrix the precipitate plate behaves like a tensile test bar.

3.4 Fe-N. - An example for a thin coherent plate is a precipitate of $\alpha^{\prime \prime} \mathrm{Fe}_{16} \mathrm{~N}_{2}$ in Fe-N alloys [28]. The transformation strain tensor is $\varepsilon=(-0.002$, $-0.002,0.097)$ and the stereogram with the corres- 
ponding cone of unextended lines is shown in figure 20 (a). The elastically soft tensile axis in the plane of unmixed strain is [100], since for iron the anisotropy ratio $A$ is greater than unity. The invariant line lies normal to this axis on the cone of unextended lines and the expected habit plane containing both directions (outlined by a great circle) is a $\left\{\begin{array}{llll}0 & 1 & 15\end{array}\right\}$ plane, $8.5^{\circ}$ inclined to the $\{001\}$ plane. A micrograph of an $\alpha^{\prime \prime}$ platelet is seen in figure $20(\mathrm{~b})$. The particles have an overall habit plane of $\{001\}$ with individual segments exhibiting different contrast due to an inclination of about $10^{\circ}$,
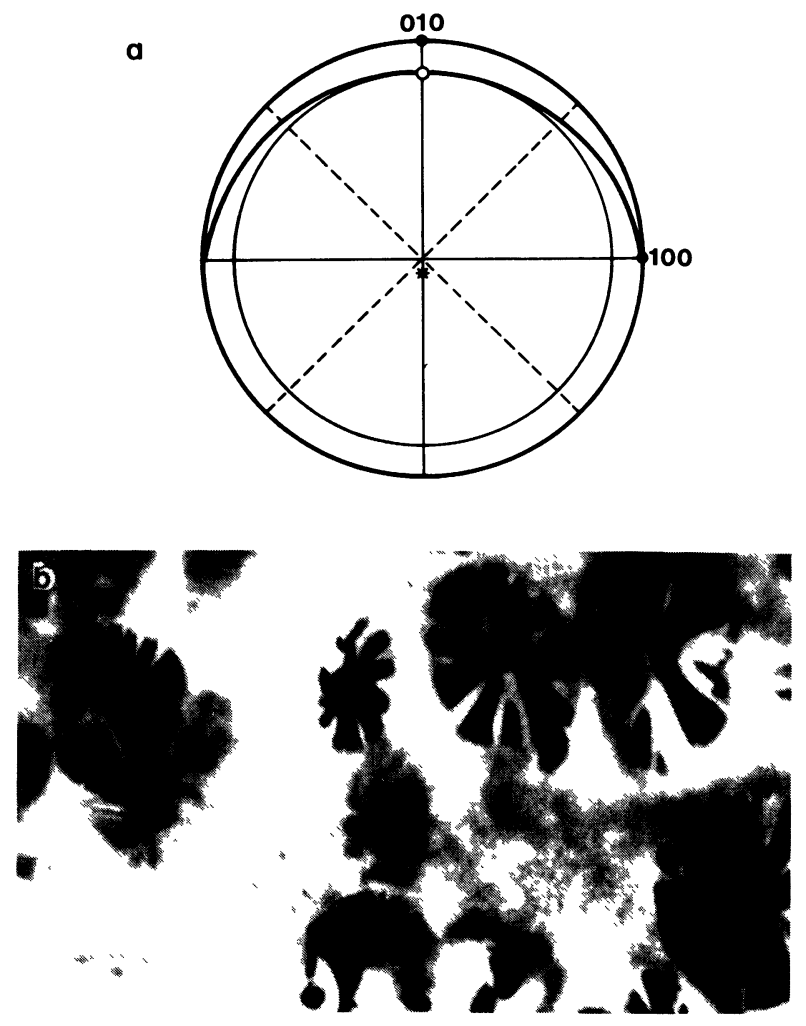

Fig. 20. - Coherent $\alpha^{\prime \prime}$ plate precipitates in Fe-N alloy (bcc-bct transformation) ; (a) shows stereogram with cone of unextended lines, elastically soft directions in the plane of unmixed strains (solid lines for $A>1$, broken lines for $A<1$ ), invariant line direction (open circle) and habit plane (star) ; (b) TEM image of actual plates made up of segments inclined as shown in (a).

near the predicted angle for an invariant line. The morphology is thus not that of a single plate but a puckered sheet made up of several mutually inclined segments. The elastic anisotropy does not appear to be a strong influence since tensile axes other than the soft [100] direction are seen in many segments.

On the other hand, the crystallographic constraints imposed by the loss of coherency exert a strong influence on the habit plane orientation of a semicoherent plate. As an example, during aging of $\mathrm{Fe}-\mathrm{N}$ alloys precipitates of the $\gamma^{\prime} \mathrm{Fe}_{4} \mathrm{~N}$ nitride with an fcc iron atom arrangement form as thin plates on well-defined habit planes [29]. The transformation
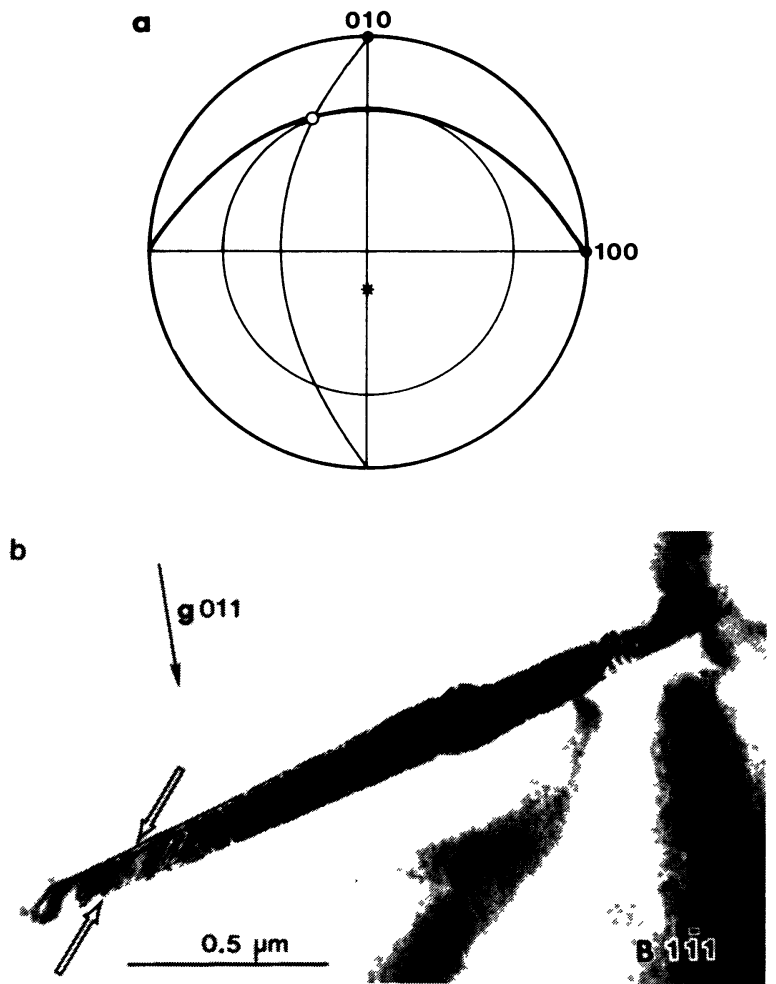

Fig. 21. - Semicoherent $\gamma^{\prime}$ plate precipitates in $\mathrm{Fe}-\mathrm{N}$ alloy (bcc-fcc transformation); (a) shows stereogram with cone of unextended lines, $(101)_{\mathrm{bcc}} /(111)_{\mathrm{fcc}}$ slip plane, [494] invariant line (open circle) and (049) habit plane (star) ; (b) shows TEM images of inclined plate with invariant line direction arrowed.

strain is $\varepsilon=(-0.064,-0.064,0.323)$, of the same form as that for $\alpha$ " precipitation but with larger strains. Figure 21(a) illustrates the cone of unextended lines and its intersection with the (101) bcc/(111) fcc slip plane in the [494] bcc invariant line direction. The tensile axis in the (001) plane of unmixed principal strains is [100] and the habit plane containing both the invariant line and the tensile axis is (049). The micrograph in figure 21(b) shows a $\gamma^{\prime}$ plate inclined to the beam with its characteristic set of striations in the interface. The direction of the striations was found to coincide with the [494] invariant line direction, and by measuring angles between different precipitate variants in edge-on orientation the habit plane was determined to be (04) 9 ).

3.5 Mo-C. - A further application of the tensile test analogy is the precipitation of $\mathrm{Mo}_{2} \mathrm{C}$ (hcp) in Mo. As shown earlier in figure 19(b) the particle interface exhibits the typical striations along a $\langle 113\rangle$ direction. The transformation strain referred to principal axes is $\varepsilon=(0.17,0.06,-0.05)$ with an elliptical cone of unextended lines (Fig. 19(a)). Slip occurs on the $(0001) \mathrm{hcp} /(110)$ bcc planes leading to an intersection with the cone at the observed [113] invariant line. The tensile axis with 
the highest resolved shear stress on the (110) plane is again [100] and the resulting habit plane is $(0 \overline{3} 1)$, shown as a great circle in figure 19(a).

3.6 Fe-C. - The precipitation of $\varepsilon$-carbide (hcp) in $\mathrm{Fe}-\mathrm{C}$ alloys is another example of a bcc-hcp transformation with a large volume change ; however, the principal strains are all of the same sign [30]. The smallest strain lies along the $\langle 010\rangle$ direction which becomes the direction of easy growth. The tensile axis is again $\langle 100\rangle$ and hence the resulting habit plane is $\{001\}$ with striations along the easy growth direction. Figure 22 shows a micrograph of $\{001\}$

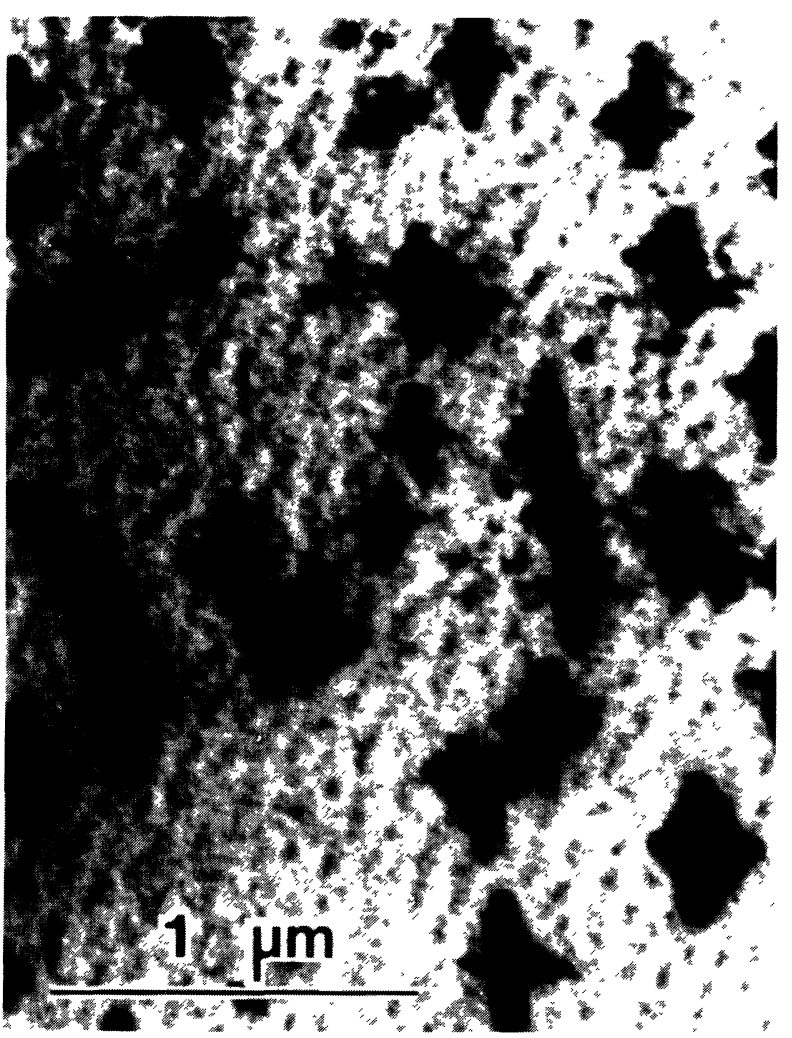

Fig. 22. - Cruciform-shaped particles of $\varepsilon$-carbide precipitated in carbon-ferrite. The $\{001\}$ plate is made up of perpendicular variants of the precipitate (micrograph by P. Ferguson).

$\varepsilon$-carbide plates in an $\mathrm{Fe}-\mathrm{C}$ alloy. Their characteristic shape is due to elongation along two orthogonal $\langle 010\rangle$ directions corresponding to two orthogonal precipitate variants in the same $\{001\}$ plane.

The good agreement between observed and predicted habit planes demonstrated in this section makes the tensile test analogy a good quantitative model for these transformations.

\section{Symmetry of precipitate shapes and distributions.}

In the previous sections we have considered the morphologies and growth mechanisms of individual precipitates. Real crystals always contain a distribu- tion of many similar precipitates. Due to crystal symmetry every particle has a number of crystallographically equivalent variants which are related to each other by symmetry elements of the matrix crystal. If nucleation is random, then all variants form with equal probability. However, an external influence such as a thermal gradient or a stress field can lead to non-random nucleation. All the examples in this article show random distributions of all equivalent variants. The reason that $\mathrm{Cr}$ needles in $\mathrm{Cu}$ (Fig. 15) appear more random than $\mathrm{Ge}$ needles in Al (Fig. 17), even though both are seen in an $\langle 001$ > zone axis, is that the $\mathrm{Cr}$ needles lie along directions of low symmetry. There are as many variants of the $\mathrm{Cr}$ precipitates, 24, as there are symmetry elements in the matrix. There are only three variants of $\mathrm{Ge}$ needles because they share many of the matrix symmetries. Thus different variants are generated only by those symmetry
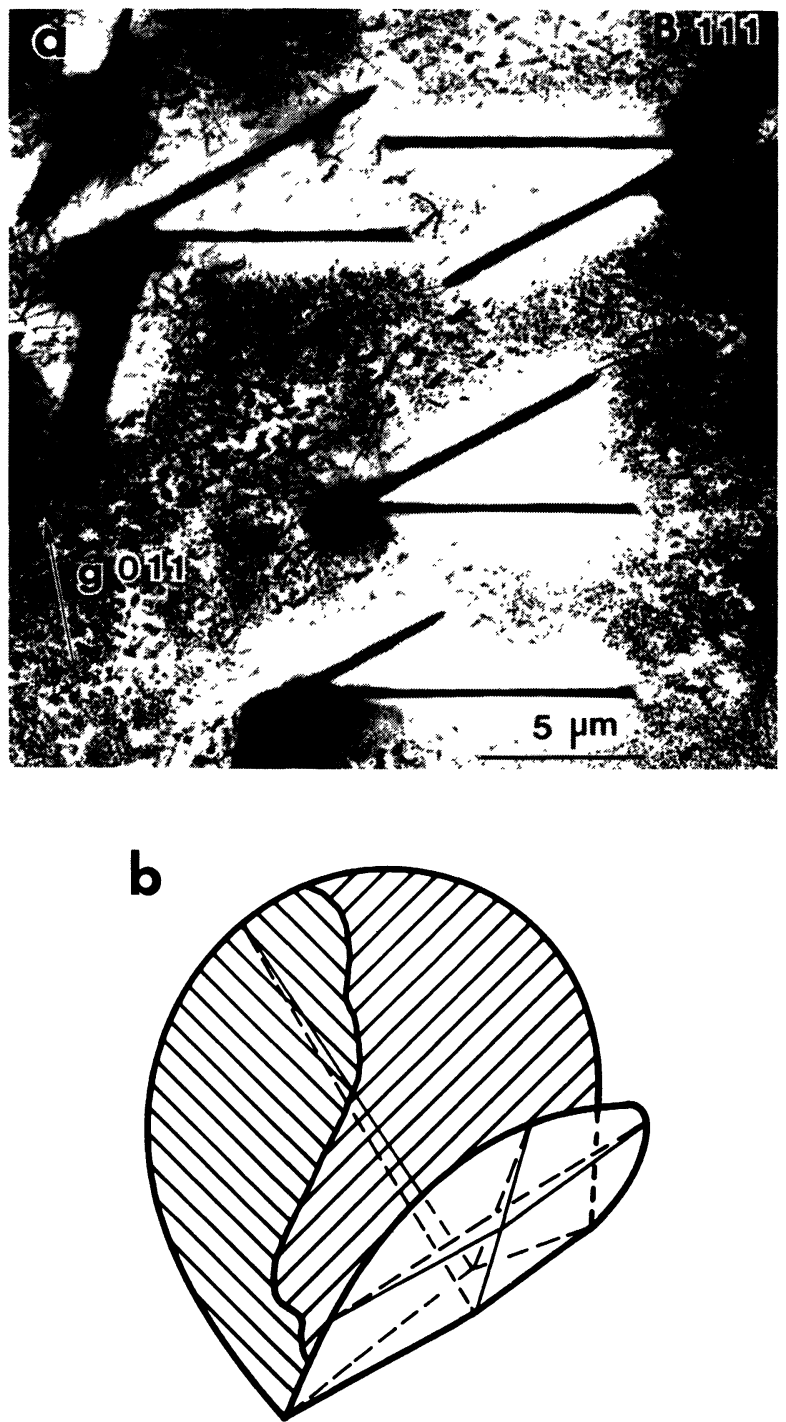

Fig. 23. - V-shaped groupings of $\gamma^{\prime}$ plates ; (a) HVEM micrograph illustrating microstructure formed by in situ aging ; (b) schematic of the arrangement of four $\gamma^{\prime}$ variants forming a $\mathrm{V}$. 
operations of the matrix that are not shared by the precipitate [31].

It is frequently found that several variants group together in a characteristic array. An example of such groups is shown in figure 23. The V-shaped particles in (a) are $\gamma^{\prime}$ precipitates seen nearly edgeon. A schematic of the entire morphology is given in (b). Each V-shaped group is in fact made up of four variants related to each other by 100 and 011 mirror planes of the matrix. Calculation of the strain field of each variant shows that the two arms of the $V$ accommodate each other's shear component. Similar self-accommodating arrays of four variants are commonly found in shape-memory alloys [32, 33].

A different kind of grouping is illustrated in the high resolution image in figure 24 which shows a

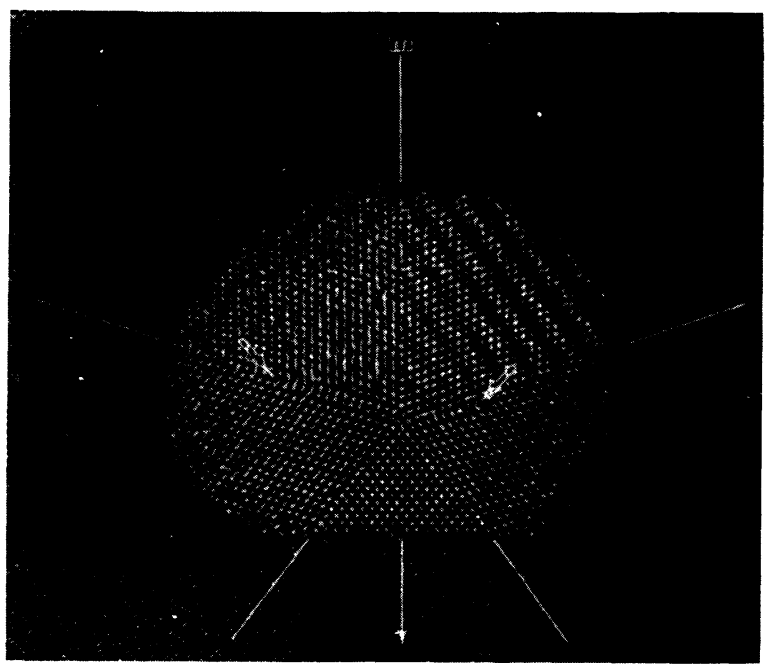

Fig. 24. - High-resolution image of multiply-twinned $\mathrm{Ge}$ particle in Al-Ge alloy seen along $\langle 001\rangle_{\mathrm{Al}} /\langle 110\rangle_{\mathrm{Ge}}$. The particle has approximately fivefold symmetry but its shape and orientation relationship are of lower $(2 \mathrm{~mm})$ symmetry.

cross-section of a $\mathrm{Ge}$ needle along $\langle 001\rangle$ in Al [34]. The five different parts of this particle exhibit three different orientation relationships. Two pairs of variants are symmetry-related across the plane marked $\mathrm{m}$ which is a mirror plane in both the matrix and the precipitate. It remains to be determined whether this fivefold group is due to strain self-accommodation or accidental internal twinning. However, it is clear that this particle, just as the other groups and single precipitates observed, hàs near-equilibrium shape conforming to the symmetry of the Wulff plot [31].

\section{Summarizing discussion.}

Considerable insight into the structural changes that occur during phase transformations can be obtained from microstructural studies of model alloy systems [35] using transmission electron microscopy at various resolution levels. Through selected examples from several different categories of transformation strain, we have attempted to highlight the structural principles underlying a precipitation reaction. All the major precipitate morphologies and orientation relationships observed can be accounted for using relatively simple concepts and models. Several basic principles can be summarized as follows: (1) the precipitate dimensions (morphology) tend to be inversely proportional to the principal strains provided they are of the same sign. (2) In systems with transformation strains of opposite signs, lattice rotations are important mechanisms for optimizing strain accommodation by forming strain-free (invariant) lines. (3) For plate-shaped precipitates, the plate orientation, i.e. the habit plane, is established in some cases by the existence of a naturally strain-free (invariant) plane, and in others by lattice slip and rotation leading to a high index habit plane with dislocations parallel to the invariant line direction. In both cases the strain energy is minimized. (4) The conditions for both coherent and semicoherent precipitate plate habit planes are similar. The optimum orientation for a coherent plate occurs so as to align a principal strain in an elastically soft direction, whereas in the semi-coherent case plastically soft directions are selected. (5) All appropriate lattice defects, elastic and plastic deformation modes, and crystal symmetries are employed by an alloy system during a precipitation reaction to accommodate the new phase in the parent matrix. As a consequence, variations in prior history, and precipitation temperature or time can result in marked changes in precipitate dispersion and morphology.

It is evident from the basic nature of these principles that they will not be restricted to simple alloy systems or to a limited range of conditions. In future work it will be interesting to apply these ideas to higher temperature conditions where non-conservative processes can occur more readily, to more complex alloy systems, and to non-metallic materials such as ceramic alloys.

\section{Acknowledgments.}

This work is supported by the Director, Office of Energy Research, Office of Basic Energy Sciences, Materials Sciences Division of the U.S. Department of Energy under Contract No. DE-AC0376SF00098. 


\section{References}

[1] Nicholson, R. B., Nutting, J., Acta Metall. 9 (1961) 332.

[2] Hren, J. J., Thomas, G., Trans. Metall. Soc. AIME 227 (1963) 308.

[3] Howe, J. M., AARonson, H. I., Gronsky, R., Acta Metall. 33 (1985) 639, 649.

[4] Christian, J. W., The Theory of transformations in Metals and Alloys, Part I, 2nd ed. (Pergamon Press, Oxford) 1975.

[5] Olson, G. B., CoHen, M., Acta Metall. 27 (1979) 1907.

[6] Howe, J. M., Gronsky, R., Proc. MRS Conf. (Boston) 1985.

[7] Dahmen, U., Westmacott, K. H., Phys. status solidi $A 80$ (1983) 249.

[8] Stobis, W. M., Purdy, G. R., Acta Metall. 26 (1978) 1069.

[9] Guyot, P., Wintenberger, M., J. Mat. Sci. 9 (1974) 614

[10] Headley, T. J., Hren, J. J., Phil. Mag. 34 (1976) 101.

[11] Dahmen, U., Westmacott, K. H., Scr. Metall. 17 (1983) 1241

[12] Witcomb, M. J., Dahmen, U., Westmacott, K. H., Acta Metall. 31 (1983) 743, 749, 755.

[13] Witcomb, M. J., Dahmen, U., Westmacott, K. H., Proc. EMSSA 11 (1981) 45.

[14] Ferguson, P., Westmacott, K. H., Fisher, R. M., Dahmen, U., Mat. Sci. Technol. 1 (1985) 53.

[15] Russell, K. C., Scr. Metall. 3 (1969) 313.

[16] Dahmen, U., Pelton, A. R., Witcomb, M. J., Westmacott, K. H., Proc. Conf. Solid-Solid Phase Transformations, ed. H. I. Aaronson (Pittsburgh) Met. Soc. AIME (1981) 637.

[17] Mrtchell, J. B., Acta Metall. 19 (1971) 1063 ; Metallography 8 (1975) 5.
[18] Westmacott, K. H., Dahmen, U., Decomposition of Alloys : the early stages, eds. P. Haasen et al. (Pergamon Press) 2nd Acta-Scripta Metall. Conf. (1984) 204.

[19] Hall, M. G., Aaronson, H. I., Kinsman, K. R., Surf. Sci. 31 (1972) 257.

[20] Weatherly, G. C., Humble, P., Borland, D., Acta Metall. 27 (1979) 1815.

[21] Dahmen, U., Ferguson, P., Westmacott, K. H., Acta Metall. 32 (1984) 803.

[22] Koster, U., Mat. Sci. Eng. 5 (1969) 174.

[23] Ozawa, E., Kimura, H., Acta Metall. 18 (1970) 995.

[24] Dahmen, U., Westmacott, K. H., Proc. MRS Conf. (Boston) 1985 in press.

[25] Lang, J. M., 40th Ann. Proc. EMSA (1982) 622.

[26] LANG, J. M., DAHMEN, U., WestmacotT, K. H., Phys. status solidi A 75 (1983) 409.

[27] Dahmen, U., Westmacott, K. H., Acta Metall. 34 (1986) 475.

[28] Ferguson, P., Dahmen, U., Westmacott, K. H., Scr. Metall. 18 (1984) 57.

[29] Booker, G. R., Acta Metall. 9 (1961) 590.

[30] Ferguson, P., DAhMEN, U., WestmacotT, K. H., to be published.

[31] Cahn, J. W., KalonjI, G., Proc. Conf. Solid-Solid Phase Transformations, ed. H. I. Aaronson (Pittsburg) Met. Soc. AIME (1981) 3.

[32] SAburi, T., Wayman, C. M., Acta Metall. 27 (1979) 979.

[33] Wadhawan, V. K., Mat. Sci. Forum 3 (1985) 91.

[34] Dahmen, U., WestmacotT, K. H., Science 233 (1986) 875.

[35] Shchegoleva, T. V., Phys. Met. Metallogr. 55 (1983) 59. 